TRANSACTIONS OF THE

AMERICAN MATHEMATICAL SOCIETY

Volume 356, Number 7 , Pages 2757-2780

S 0002-9947(03)03435- 4

Article electronically published on December 15, 2003

\title{
DYNAMICAL APPROACH TO SOME PROBLEMS IN INTEGRAL GEOMETRY
}

\author{
BORIS PANEAH
}

\begin{abstract}
As is well known, the main problem in integral geometry is to reconstruct a function in a given domain $D$, where its integrals over a family of subdomains in $D$ are known. Such a problem is interesting not only as an object of pure analysis, but also in connection with various applications in practical disciplines. The most remarkable example of such a connection is the Radon problem and tomography. In this paper we solve one of these problems when $D$ is a bounded domain in $\mathbb{R}^{2}$ with a piecewise smooth boundary. Some intermediate results related to dynamical systems with two generators and to some functional-integral equations are new and interesting per se. As an application of the results obtained we briefly study a boundary problem for a general third order hyperbolic partial differential equation in a bounded domain $D \subset \mathbb{R}^{2}$ with data on the whole boundary $\partial D$.
\end{abstract}

\section{INTRODUCTION}

This paper is mainly devoted to one of the typical problems in integral geometry: to reconstruct a function in a domain $D$, where its integrals over a family of subdomains in $D$ are known. A peculiarity of the present work is that we consider bounded domains $D$ with boundary $\partial D$. The statement of the problem under consideration and the results we obtain turn out to be intimately connected with both local and global properties of $\partial D$. Roughly speaking, given a connected bounded domain $D \subset \mathbb{R}^{2}$, let $D_{q}$ be a system of subdomains in $D$, parameterized by points $q \in \partial D$. To any function $f$ in $\bar{D}$ we associate the integrals

$$
\int_{D_{q}} f d \sigma=h(q), \quad q \in \partial D .
$$

In connection with this relation, the above-mentioned problem in integral geometry can be formulated in the following way (see [1]): for what spaces of functions $f$ and $h$ is the map $f \mapsto h$ one-to-one, and what functions $h(q)$ can be represented by the integral (1)?

In this paper we investigate the situation in which $D$ is a curvilinear plane triangle. Even in this simplest case there are many candidates for the roles of the above spaces. We restrict ourselves here to a class of spaces which appears in studying boundary problems for some hyperbolic differential equations in suitable bounded domains (see Section 7). In a framework of this class of function spaces we give an exhaustive answer to the above questions. With each of these function

Received by the editors February 8, 2002 and, in revised form, January 28, 2003.

2000 Mathematics Subject Classification. Primary 45E99, 39B22; Secondary 37E99, 35L35. 
spaces we associate a noncommutative semigroup of maps in $\partial D$. This semigroup naturally generates a noncommutative dynamical system with a set $\mathcal{O}$ of specific orbits in $\partial D$. As we will show, the solvability of equation (1) and the uniqueness of a solution depend only on whether the set $\mathcal{O}$ contains periodic orbits of a special type. Every such orbit, if any, plays the role of an obstruction in constructing a solution of equation (1). It is worth mentioning that the corresponding conditions of solvability are absolutely transparent from the geometric point of view.

In obtaining the main results we transform relation (1) into some functionalintegral equation on a part $\Gamma$ of the boundary $\partial D$. In a simplest situation this equation has the form

$$
F-a_{1} F \circ \delta_{1}-a_{2} F \circ \delta_{2}=H,
$$

where $a_{1}, a_{2}$ and $H$ are given real-valued continuous functions on $\Gamma, \delta_{1}$ and $\delta_{2}$ are continuous maps of $\Gamma$ into itself, and $F$ is an unknown function on $\Gamma$. Such functional equations have never been investigated before; they are interesting by themselves and may be applied in other fields of analysis (see [4]). We derive an essential piece of information related to the solvability of equation (2) and to qualitative properties of its solutions by means of dynamical methods. An application of these methods becomes possible if we associate this equation with a semigroup $\Phi_{\delta}$ of maps of $\Gamma$ into itself generated by $\delta_{1}$ and $\delta_{2}$. In terms of some specific orbits of this semigroup a necessary and sufficient condition for the existence of a unique solution to equation (2) is formulated. In proving corresponding assertions we obtain, in particular, a maximum principle for the functional equations in question.

As one of the applications of the obtained results we consider a new type of boundary problem for a class of higher $(>2)$ order hyperbolic differential equations in a bounded domain (see Section 7). The main result related to the wellposedeness of this problem is formulated, and we prove the corresponding statement by reducing the boundary problem in question to equation (1).

Some preliminary results are contained in the author's papers [2], [4]

\section{Statement of The PROBlem AND DEFinitions}

2.1. Let $\boldsymbol{l}_{1}$ and $\boldsymbol{l}_{2}$ be smooth nonsingular transversal vector fields in a disk $B$ in the space $\mathbb{R}^{2}$ of the variable $y=\left(y_{1}, y_{2}\right)$. Introduce a curvilinear triangle $D=O A_{1} A_{2}$ whose sides $O A_{1}$ and $O A_{2}$ are trajectories of the vector fields $\boldsymbol{l}_{1}$ and $\boldsymbol{l}_{2}$, respectively. As to the side $\Gamma=A_{1} A_{2}$, it is assumed to be an arbitrary smooth curve without

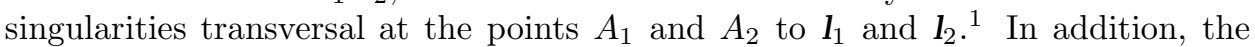
closure $\bar{D}$ of a domain $D$ is supposed to satisfy the following hypotheses:

$1^{\circ}$ For any point $p \in \bar{D}$ the trajectory of $\boldsymbol{l}_{j}$ passing through $p$ meets $O A_{k}, j, k=$ $1,2, j \neq k$, at a point $\pi_{k} p$.

$2^{\circ}$ The set $\bar{D}$ is $\boldsymbol{l}_{j}$-convex, $j=1,2$. This means that, given points $p$ and $q$ on any trajectory $\gamma_{j}$ of the field $\boldsymbol{l}_{j}$, all the points $r \in \gamma_{j}$ between $p$ and $q$ belong to $\bar{D}$.

Given an arbitrary point $q \in \Gamma$, let $D_{q}$ be a curvilinear parallelogram $q q_{1} O q_{2}$, where $q_{j}=\pi_{j} q, j=1,2$. The above conditions $1^{\circ}$ and $2^{\circ}$ guarantee an inclusion $\bar{D}_{q} \subset \bar{D}$ for all $q \in \Gamma$.

\footnotetext{
1 See Figure 1.
} 
In this work we study the solvability of an integral equation of the following form:

$$
\int_{D_{q}} f d \sigma=h(q), \quad q \in \Gamma .
$$

Here $\sigma$ is a measure in $B, h(q) \in C(\Gamma)$ is a given function and $f \in C(\bar{D})$ is an unknown function. The analogy of problem (3) with the famous Radon problem is obvious.

Remark. Note that the problem in question is insensitive to multiplying the vector fields $\boldsymbol{l}_{1}$ and $\boldsymbol{l}_{2}$ by any nonvanishing functions $p_{1}$ and $p_{2}$, respectively.

It can be verified directly that the range of the operator

$$
\mathcal{B}: f \in C(\bar{D}) \rightarrow \int_{D_{q}} f d \sigma \in C(\Gamma)
$$

is (a part of) a linear space

$$
\mathcal{H}(\Gamma)=\left(C^{2} \cap C_{0}\right)(\Gamma)
$$

of all twice continuously differentiable in $\Gamma$ functions vanishing at the boundary points of $\Gamma$. Therefore, the best possible solution of the problem, as formulated in the Introduction, consists in describing spaces $\mathcal{F}(D) \subset C(\bar{D})$ for which the map

$$
\mathcal{B}: \mathcal{F}(D) \rightarrow \mathcal{H}(\Gamma)
$$

is one-to-one. Among various candidates for the role of $\mathcal{F}(D)$ we have chosen a wide class of subspaces in $C(\bar{D})$ which naturally appear in the theory of boundary problems for PDE.

Definition. Given a smooth nonsingular vector field $\boldsymbol{l}$ in $B$ we denote by $C_{\langle l\rangle}(D)$ the subset of all functions in $C(\bar{D})$ which remain constant along each trajectory of the field 1 .

It is not difficult to describe the space $C_{\langle 1\rangle}(D)$ directly. Let

$$
\mathbf{I}=\left\{\lambda_{1}(y), \lambda_{2}(y)\right\}, \quad y=\left(y_{1}, y_{2}\right) \in B,
$$

be a coordinate form of the vector field 1 . Denote by $\omega(y)$ a smooth function without critical points which solves the first order differential equation

$$
\lambda_{1}(y)\left(\partial / \partial y_{1}\right) \omega+\lambda_{2}(y)\left(\partial / \partial y_{2}\right) \omega=0 .
$$

Then the space $C_{\langle I\rangle}(D)$ consists of all functions $f(y)=F(\omega(y))$, with $F$ being an arbitrary real-valued continuous function on the range of $\omega$.

2.2. Let $\boldsymbol{l}$ be a smooth nonsingular vector field in $B$ such that

(i) $\boldsymbol{l}$ is transversal to $\Gamma$ and to both fields $\boldsymbol{l}_{1}$ and $\boldsymbol{l}_{2}$;

(ii) for any point $p \in \bar{D}$ a trajectory of $\boldsymbol{l}$ passing through $p$ meets the curve $\Gamma$ at a point $\pi_{l} p$.

By virtue of hypothesis (ii), we can introduce two maps in $\Gamma$ :

$$
\zeta_{1}=\pi_{1} \circ \pi_{1} \quad \text { and } \quad \zeta_{2}=\pi_{1} \circ \pi_{2}
$$

which play a crucial role in formulating main results of the work. These maps generate a noncommutative semigroup $\Phi_{\zeta}$. The elements of $\Phi_{\zeta}$ are all the maps of $\Gamma$ into itself of the form

$$
\zeta_{J}=\zeta_{j_{n}} \circ \ldots \circ \zeta_{j_{1}}
$$


where $J=\left(j_{1}, \ldots, j_{n}\right)$ is a multi-index with $j_{k}$ equal to 1 or 2 , and $\circ$ denotes the composition of maps. The semigroup $\Phi_{\zeta}$ naturally determines a dynamical system of a cascade type. In what follows, we use the geometric terminology relating to $\Phi_{\zeta}$ and not coinciding completely with the traditional one.

1) Given a map $\zeta_{J} \in \Phi_{\zeta}$, an ordered set $\mathcal{O}=\left(q_{1}, \ldots, q_{n+1}\right)$ of points in $\Gamma$ is called an orbit if

$$
q_{k+1}=\zeta_{j_{k}}\left(q_{k}\right) \text { for } 1 \leq k \leq n \leq \infty .
$$

The set of all orbits will be denoted by $\mathcal{O}_{\zeta}$.

Introduce the guiding set:2

$$
\mathcal{T}_{1}=\left\{q \in \Gamma \mid \mathbf{l}_{1}(q) \in T_{q}(\Gamma)\right\}, \quad \mathcal{T}_{2}=\left\{q \in \Gamma \mid \mathbf{l}_{2}(q) \in T_{q}(\Gamma)\right\}, \quad \text { and } \mathcal{T}=\mathcal{T}_{1} \cup \mathcal{T}_{2},
$$

and denote by $\mathcal{T}_{1}^{\prime}, \mathcal{T}_{2}^{\prime}$ and $\mathcal{T}^{\prime}$ the collections of all limit points of these sets, respectively.

2) An orbit $\mathcal{O}=\left(q_{1}, \ldots, q_{n+1}\right)$ is said to be $\mathcal{T}$-proper if

$$
\zeta_{j_{k}}=\zeta_{1} \quad \text { for } q_{k} \in \mathcal{T}_{1} \quad \text { and } \quad \zeta_{j_{k}}=\zeta_{2} \quad \text { for } q_{k} \in \mathcal{T}_{2}
$$

in (5).

3) If all points of an orbit $\mathcal{O}$ belong to a guiding set $\mathcal{T}$, then $\mathcal{O}$ is called a $\mathcal{T}$-guided orbit.

4) If $q_{1}=q_{n+1}$ or, equivalently, if $\zeta_{J}\left(q_{1}\right)=q_{1}$, then the orbit $\mathcal{O}=\left(q_{1}, \ldots, q_{n+1}\right)$ is called a periodic orbit or a cycle.

From the point of view of the dynamical system generated by the semigroup $\Phi_{\zeta}$, in moving along any $\mathcal{T}$-proper orbit we leave each point $q_{j} \in \mathcal{T}$ along a trajectory transversal to $\Gamma$.

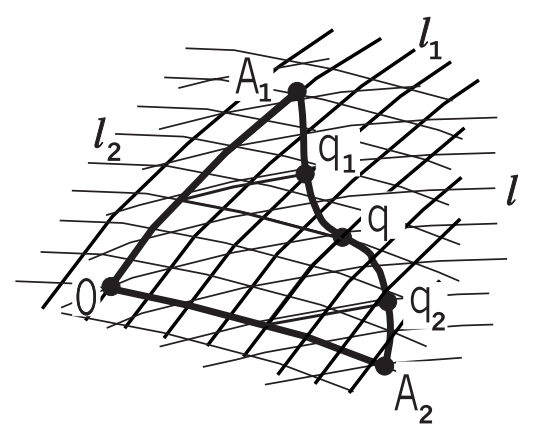

Figure 1. The families of trajectories of the vector fields $\boldsymbol{l}_{1}, \boldsymbol{l}_{2}$ and $\boldsymbol{I}$ are represented. The point $q \in \Gamma$ is in $\mathcal{T}_{2}$. Therefore, the orbit $\left(q, q_{2}\right)$ is $\mathcal{T}$-proper whereas the orbit $\left(q, q_{1}\right)$ is not.

Definition. We denote by $\mathfrak{N}_{\zeta}^{\mathcal{T}}$ the set of all $\mathcal{T}$-proper $\mathcal{T}$-guided periodic orbits in $\Gamma$.

\footnotetext{
${ }^{2}$ As usual, $T_{q}(\Gamma)$ stands for the tangent space of the curve $\Gamma$ at the point $q$.
} 


\section{The MAIN RESUlts}

3.1. Before formulating the main results we note that, by virtue of hypotheses $1^{\circ}$ and $2^{\circ}$ in Subsection 2.1, there is a neighborhood $V$ of the curvilinear triangle $D$ in the space $\mathbb{R}^{2}$ of the variable $y=\left(y_{1}, y_{2}\right)$ and a diffeomorphism $y: W \ni x \rightarrow$ $y(x) \in V$ of a domain $W$ in the space $\mathbb{R}^{2}$ of the variable $x=\left(x_{1}, x_{2}\right)$ satisfying the following conditions:

(I) $\quad D$ is the image of a curvilinear triangle $\widetilde{D}$ whose sides are the intervals $\left\{x_{1} \mid 0 \leq x_{1} \leq 1\right\}$ and $\left\{x_{2} \mid 0 \leq x_{2} \leq 1\right\}$ of the $x_{1}$ - and $x_{2}$-axis, respectively, and a smooth curve $\widetilde{\Gamma}$.

(II) All trajectories of the fields $\boldsymbol{l}_{1}$ and $\boldsymbol{l}_{2}$ are $y$-images of trajectories of the unit vector fields $\boldsymbol{e}_{1}$ and $\boldsymbol{e}_{2}$ parallel to the $x_{1}$ - and $x_{2}$-axis, respectively.

In particular, it follows that for certain smooth positive functions $p_{1}(y)$ and $p_{2}(y)$ the relations

$$
\left(\partial / \partial x_{1}\right) y=p_{1} \boldsymbol{l}_{1}(y), \quad\left(\partial / \partial x_{2}\right) y=p_{2} \boldsymbol{l}_{2}(y)
$$

hold for all $x \in \widetilde{D}$. On the other hand, it is clear that the triangle $\widetilde{D}$ satisfies the same hypotheses $1^{\circ}$ and $2^{\circ}$ (see Subsection 2.1) in which the fields $\boldsymbol{e}_{j}$ are substituted for $\boldsymbol{l}_{j}, j=1,2$.

In Theorem 1 and Theorem 2 below the measure $d \sigma$ in equation (3) is defined as

$$
d \sigma=d y_{1} d y_{2} /\left|p_{1} \boldsymbol{l}_{1}(y) \times p_{2} \boldsymbol{I}_{2}(y)\right| .
$$

3.2. We are now able to state the main results of this work.

Theorem 1. Let $\boldsymbol{I}=\lambda_{1} p_{1} \boldsymbol{l}_{1}+\lambda_{2} p_{2} \boldsymbol{l}_{2}$ be a vector field in $B$, where $\lambda_{1}$ and $\lambda_{2}$ are certain positive constants. Assume that either at least one of the sets $\mathcal{T}_{1}^{\prime}$ and $\mathcal{T}_{2}^{\prime}$ is finite or all possible pairs of points $\tau_{1} \in \mathcal{T}_{1}^{\prime}$ and $\tau_{2} \in \mathcal{T}_{2}^{\prime}$ are situated on $\Gamma$ in the order $A_{1}, \tau_{1}, \tau_{2}, A_{2}$. Then for an arbitrary function $h \in \mathcal{H}(\Gamma)$ there is a unique solution $f \in C_{\langle 1\rangle}(D)$ of equation (3) if and only if

$$
\mathfrak{N}_{\zeta}^{\mathcal{T}}=\emptyset .
$$

The inverse operator $\mathcal{B}^{-1}: h \mapsto f$ is a continuous operator from $\mathcal{H}(\Gamma)$ to $C_{\langle I\rangle}(D)$.

To illustrate this result let us consider Figures 2 and 3 . In both figures $p$ is the only point in $\Gamma$ belonging to $\mathcal{T}_{1}$ and $q$ is the only point in $\Gamma$ belonging to $\mathcal{T}_{2}$. Therefore, the only orbit consisting of the points $p$ and $q$ may belong to $\mathfrak{N}_{\zeta}^{\mathcal{T}}$. In Figure 2 a unique $\mathcal{T}$-proper orbit beginning at the point $p$ (q, respectively) contains as a second one the point $p_{1} \notin \mathcal{T}\left(q_{2} \notin \mathcal{T}\right.$, respectively). Thus, $\mathfrak{N}_{\zeta}^{\mathcal{T}}=\emptyset$, and problem (3) is uniquely solvable in the triangle $O A_{1} A_{2}$ for all functions $h \in \mathcal{H}(\Gamma)$. In Figure $3 \mathfrak{N}_{\zeta}^{\mathcal{T}} \neq \emptyset$ as the orbit $(p, q, p)$ is obviously in $\mathfrak{N}_{\zeta}^{\mathcal{T}}$. In view of Theorem 1 (see relation (44) below), the equation $\mathcal{B} f=h$ is not solvable if

$$
\partial_{s} h(p)+\partial_{s} h(q) \neq 0,
$$

where $\partial_{s}$ is the differentiation with respect to the natural parameter on $\Gamma$.

Our next result relates to the solvability properties of equation (3) in the case of the same field $l$, but with variable coefficients $\lambda_{1}$ and $\lambda_{2}$. 


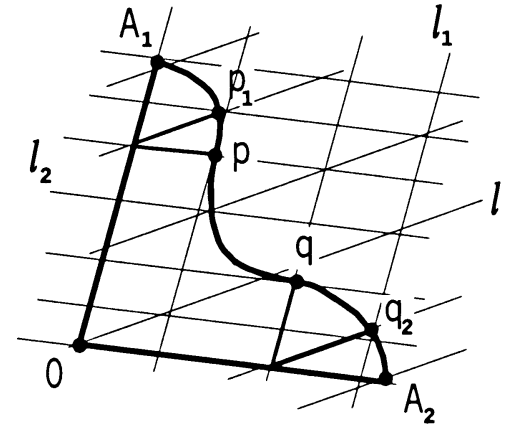

FiguRe 2.

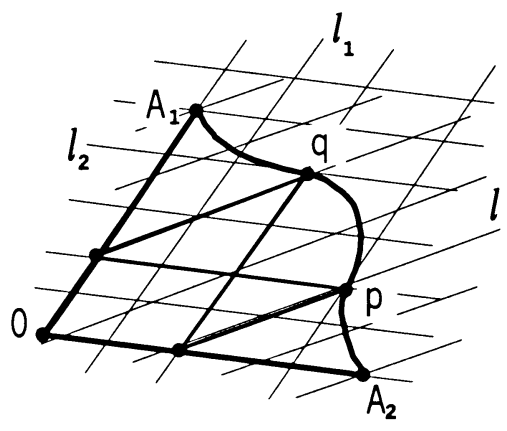

Figure 3.

Theorem 2. Assume that a vector field $\mathbf{l}=\lambda_{1} p_{1} \boldsymbol{l}_{1}+\lambda_{2} p_{2} \boldsymbol{l}_{2}$ satisfies the following conditions:

$$
\begin{array}{ll}
\left(\partial / \partial \mathbf{l}_{1}\right) \lambda_{1}=0, & \left(\partial / \partial \mathbf{l}_{2}\right) \lambda_{2}=0 \\
\left(\partial / \partial \mathbf{l}_{2}\right) \lambda_{1} \leq 0, & \left(\partial / \partial \mathbf{l}_{1}\right) \lambda_{2} \leq 0 .
\end{array}
$$

If at least one of the sets $\mathcal{T}_{1}^{\prime}$ and $\mathcal{T}_{2}^{\prime}$ is finite, then the conclusions of Theorem 1 are valid, provided that $\mathfrak{N}_{\zeta}^{\mathcal{T}}=\emptyset$.

Passing to the concluding Theorem 3 we note that from the technical point of view this is the central part of the work. We continue to discuss the solvability properties of equation (3) but in the simplest (one can say, in a model) situation: both of the vector fields $\boldsymbol{l}_{1}$ and $\boldsymbol{l}_{2}$ are constant, while $d \sigma=d y_{1} d y_{2}$. However, the vector field $\boldsymbol{l}$ in question is supposed to be of a rather general form. This makes it possible to use Theorem 3 in searching various classes of fields 1 guaranteeing the existence of the above operator $\mathcal{B}^{-1}$ and its continuity. In particular, an essential part of the above Theorem 1 and Theorem 2 is proved below by referring to Theorem 3.

Theorem 3. (I) Let $\boldsymbol{I}_{1}$ and $\boldsymbol{I}_{2}$ be the unit vector fields in the space $\mathbb{R}^{2}$ parallel to the coordinate $x_{1}$ - and $x_{2}$-axes, respectively, and let one of the sets $\mathcal{T}_{1}^{\prime}$ and $\mathcal{T}_{2}^{\prime}$ be finite. Assume that a vector field $\mathbf{l}=\lambda_{1}(x) \boldsymbol{I}_{1}+\lambda_{2}(x) \boldsymbol{l}_{2}$ with positive $\lambda_{j}(x)$ satisfies the following conditions:

$$
\begin{array}{ll}
1^{\circ} & \left(\partial / \partial x_{1}\right)\left(\lambda_{1} / \lambda_{2}\right) \geq 0, \quad\left(\partial / \partial x_{2}\right)\left(\lambda_{2} / \lambda_{1}\right) \geq 0 \quad \text { in } \quad D ; \\
2^{\circ} & \text { there is a solution } \omega \text { of equation }(4) \text { satisfying the condition } \\
& \partial_{j} \omega \partial_{j}^{2} \omega \leq 0 \quad \text { in } D \quad \text { for } j=1,2 .
\end{array}
$$

Then, under hypothesis $\mathfrak{N}_{\zeta}^{\mathcal{T}}=\emptyset$, for any function $h \in \mathcal{H}(\Gamma)$ equation (3) with $d \sigma=d x_{1} d x_{2}$ has a unique solution $f \in C_{\langle I\rangle}(D)$.

(II) If the coefficients $\lambda_{1}$ and $\lambda_{2}$ are constants and the guiding sets $\mathcal{T}_{1}$ and $\mathcal{T}_{2}$ satisfy the hypothesis in Theorem 1, then for an arbitrary function $h \in \mathcal{H}(\Gamma)$ equation (3) with $d \sigma=d x_{1} d x_{2}$ is uniquely solvable in $C_{\langle 1\rangle}(D)$ if and only if $\mathfrak{N}_{\zeta}^{\mathcal{T}}=\emptyset$.

All these assertions will be proved in the subsequent sections. 


\section{Proof of Theorem 3}

The proof of Theorem 3 is long and consists of three parts. At first we reduce problem (1) in equivalent manner to two different functional-integral equations on the curve $\Gamma$. For the first one we obtain a maximum principle from which the uniqueness of a solution to problem (3) (i.e. the relation $\operatorname{dim} \operatorname{ker} \mathcal{B}=0$ ) follows immediately. As to the second equation on $\Gamma$, we prove that the spectral radius of the corresponding linear operator in $C(\Gamma)$ is less than one. This makes it possible to apply the Riesz-Schauder theory, and to establish that the index of this operator equals zero. Therefore, ind $\mathcal{B}=0$. Combining this result with the uniqueness already proved completes the proof of Theorem 3 .

4.1. Reduction of problem (3) to functional-integral equations. Without loss of generality we can consider the domain $D$ as a curvilinear triangle whose sides are intervals $0 \leq x_{1} \leq 1$ and $0 \leq x_{2} \leq 1$ of the $x_{1}$ - and $x_{2}$-axis, respectively, and a curve

$$
\Gamma=\left\{\left(x_{1}, x_{2}\right) \mid x_{1}=\alpha_{1}(z), \quad x_{2}=\alpha_{2}(z) ; \quad z \in I_{Z}\right\}
$$

Here

$$
I_{Z}=\{z \mid-1 \leq z \leq 1\},
$$

and $\alpha_{1}(z), \alpha_{2}(z)$ are smooth functions such that

$$
\left|\alpha_{1}^{\prime}(z)\right|+\left|\alpha_{2}^{\prime}(z)\right|>0, \quad z \in I_{Z}
$$

We note that

$$
\alpha_{1}(-1)=0, \quad \alpha_{2}(-1)=-1, \quad \alpha_{1}(1)=1, \quad \alpha_{2}(1)=0,
$$

and

a point $\left(\alpha_{1}\left(z_{0}\right), \alpha_{2}\left(z_{0}\right)\right) \in \Gamma$ belongs to a guiding set $\mathcal{T}_{j}, j=1,2$,

if and only if

$$
\alpha_{j^{\prime}}^{\prime}\left(z_{0}\right)=0, \quad j^{\prime} \neq j
$$

Introduce the notation

$$
\alpha(z)=\left(\alpha_{1}(z), \alpha_{2}(z)\right), \quad z \in I_{Z} .
$$

It is convenient to treat $\alpha$ as a map

$$
\alpha: I_{Z} \ni z \rightarrow \alpha(z) \in \Gamma
$$

which is invertible, due to (9). We observe also that, by virtue of our assumptions about the domain $D$ (see $1^{\circ}$ and $2^{\circ}$ in Subsection 2.1), the inequalities

$$
\alpha_{1}^{\prime}(z) \geq 0 \quad \text { and } \quad \alpha_{2}^{\prime}(z) \leq 0, \quad z \in I_{Z}
$$

hold. (This fact is precisely what makes it possible to translate an invariant geometric description of the above domain $D$ into an analytic language.) We will prove these inequalities (which are not quite trivial) in the Appendix.

Let $\omega=\omega(x)$ be an (arbitrary) fixed solution of equation (4) such that grad $\omega \neq 0$ in $\bar{D}$. As $\lambda_{1}>0$ and $\lambda_{2}>0$, it follows from (4) that $\left(\omega_{x_{1}} \omega_{x_{2}}\right)(x)<0$ in $\bar{D}$. Therefore one can assume (multiplying $\omega$ by -1 if it is necessary) that

$$
\omega_{x_{1}}(x)>0, \quad \omega_{x_{2}}(x)<0 \quad \text { in } \bar{D} .
$$

Introduce notation

$$
\gamma_{-}=\inf _{D} \omega, \quad \gamma_{+}=\sup _{D} \omega
$$


and let $I_{T}=\left\{t \mid \gamma_{-} \leq t \leq \gamma_{+}\right\}$. Denote by $\omega_{\Gamma}$ the restriction of the function $\omega$ to $\Gamma$. We will prove now that the map $\omega_{\Gamma}: \Gamma \rightarrow I_{T}$ is surjective and invertible. To this end consider a map

$$
\Omega=\omega \circ \alpha: I_{Z} \rightarrow I_{T} .
$$

Differentiating the function $\Omega(z)=\omega(\alpha(z))$ and using inequalities (9), (11) and (12) we arrive at the inequality

$$
\Omega^{\prime}(z)>0, \quad z \in I_{Z} .
$$

This results in invertibility of $\Omega$. But $\omega \circ \alpha=\omega_{\Gamma} \circ \alpha$ and, by virtue of invertibility of $\alpha$, the same is true with respect to the map $\omega_{\Gamma}$. To prove the surjectiveness of $\omega_{\Gamma}$ take a point $p_{-} \in \bar{D}$ such that $\omega\left(p_{-}\right)=\gamma_{-}$. Then a trajectory of the vector field $\boldsymbol{l}$ passing through $p_{-}$meets $\Gamma$ at a point $q_{-}$(see hypothesis (ii) in Section 2). Therefore $\omega_{\Gamma}\left(q_{-}\right)=\gamma_{-}$. In just the same way we determine a point $q_{+} \in \Gamma$ such that $\omega_{\Gamma}\left(q_{+}\right)=\gamma_{+}$. Thus

$$
\gamma_{-}=\min \omega_{\Gamma} \quad \text { and } \quad \gamma_{+}=\max \omega_{\Gamma}
$$

and this completes the proof of the assertion.

We return to the definitions in Section 2 and note that

$$
\pi_{1}(x)=x_{1}, \quad \pi_{2}(x)=x_{2}
$$

for any point $x=\left(x_{1}, x_{2}\right)$ in $\Gamma$. By the definition of $\omega$ and $\zeta_{j}$ we have

$$
\omega\left(x_{1}, 0\right)=\omega\left(\zeta_{1}(x)\right) \quad \text { and } \omega\left(0, x_{2}\right)=\omega\left(\zeta_{2}(x)\right) .
$$

Denote

$$
\omega_{1}(x)=\omega\left(x_{1}, 0\right), \quad \omega_{2}(x)=\omega\left(0, x_{2}\right) .
$$

Then

$$
\omega_{j}(\alpha(z))=\omega_{\Gamma}\left(\zeta_{j} \circ \alpha(z)\right), \quad j=1,2 .
$$

Introduce the smooth maps in $I_{T}$

$$
\delta_{j}=\omega_{j} \circ \alpha \circ \Omega^{-1}, \quad j=1,2,
$$

which play an important role in the following. Recalling that $\Omega=\omega \circ \alpha$ and using (14) we conclude that

$$
\delta_{j}=\omega_{\Gamma} \circ \zeta_{j} \circ \omega_{\Gamma}^{-1}, \quad j=1,2,
$$

and

$$
\omega_{j} \circ \alpha=\delta_{j} \circ \Omega .
$$

All needed properties of the maps $\delta_{j}$ are collected in the following lemma.

Lemma 4. (i) Both maps $\delta_{1}$ and $\delta_{2}$ in $I_{T}$ are nondecreasing functions. In addition $\delta_{1}$ maps $I_{T}$ onto $\left[\gamma_{0}, \gamma_{+}\right]$and $\delta_{2}$ maps $I_{T}$ onto $\left[\gamma_{-}, \gamma_{0}\right]$, where $\gamma_{0}=\omega(0,0)$.

(ii) For all values $t, \gamma_{-}<t<\gamma_{+}$, the inequalities

$$
\delta_{2}(t)<t<\delta_{1}(t)
$$

hold. Furthermore, $\delta_{1}\left(\gamma_{+}\right)=\gamma_{+}$and $\delta_{2}\left(\gamma_{-}\right)=\gamma_{-}$.

(iii) For any $t \in I_{T}$ the inequality

$$
\delta_{1}^{\prime}(t)+\delta_{2}^{\prime}(t)>0
$$

holds. 
Proof. (i) We note first of all that

$$
(d / d z) \omega_{j}(\alpha(z))=\omega_{x_{j}}\left(\pi_{j}(\alpha(z))\right) \circ \alpha_{j}^{\prime}(z) .
$$

Combining (11) and (12) results in the inequality $(d / d z)\left(\omega_{j} \circ \alpha\right)(z) \geq 0$. This inequality along with (13) and (16) leads to the monotonicity of $\delta_{j}$. Furthermore, in order to describe the images of the maps $\delta_{j}$ it suffices to note that, by virtue of $(13)$,

$$
\Omega^{-1}\left(\gamma_{-}\right)=-1, \quad \Omega^{-1}\left(\gamma_{+}\right)=1,
$$

and to verify directly that

$$
\omega_{1}(\alpha(1))=\gamma_{+}, \quad \omega_{2}(\alpha(-1))=\gamma_{-}, \quad \text { and } \quad \omega_{1}(\alpha(-1))=\omega_{2}(\alpha(1))=\gamma_{0} .
$$

(ii) Inequalities

$$
\omega_{2}(\alpha(z))<\omega(\alpha(z))<\omega_{1}(\alpha(z))
$$

hold due to (12). Substituting $\Omega^{-1}(t)$ for $z$ results in (17).

(iii) Differentiating (16) results in

$$
\omega_{x_{j}}\left(\pi_{j} \circ \alpha(z)\right) \alpha_{j}^{\prime}(z)=\left(\delta_{j}^{\prime} \circ \Omega\right)(z) \Omega^{\prime}(z)
$$

and it remains to use inequalities (12), (13) and (9).

Analogously to Section 2 one can construct a semigroup $\Phi_{\delta}$ of maps in $I_{T}$ of the form $\delta_{J}=\delta_{j_{1}} \circ \ldots \circ \delta_{j_{n}}$ with $J=\left(j_{1}, \ldots, j_{n}\right)$ and all $j_{k}$ equal to 1 or 2 . Each map $\delta_{J}$ generates an orbit consisting of points $\left(t_{1}, t_{2}, \ldots, t_{n+1}\right)$ in $I_{T}, n \geq 1$, where

$$
t_{k+1}=\delta_{j_{k}}\left(t_{k}\right), \quad k \geq 1 .
$$

We denote the union of such orbits over all multi-indices $J$ by $\mathcal{O}_{\delta}$.

By virtue of (15), we conclude that for an arbitrary multi-index $J$

$$
\delta_{J}=\omega_{\Gamma} \circ \zeta_{J} \circ \omega_{\Gamma}^{-1} .
$$

Analogously to Subsection 2.2, we introduce the guiding sets $\mathcal{T}_{\delta_{j}}=\left\{t \in I_{T} \mid\right.$ $\left.\delta_{j^{\prime}}^{\prime}(t)=0\right\}$, where $1 \leq j, j^{\prime} \leq 2$ and $j \neq j^{\prime}$. Let $\mathcal{T}_{\delta}=\mathcal{T}_{\delta_{1}} \cup \mathcal{T}_{\delta_{2}}$. An orbit $\mathcal{O}=\left(t_{1}, t_{2}, \ldots, t_{n+1}\right)$ in $I_{T}$ is called $\mathcal{T}_{\delta}$-proper if in $(20)$

$$
\delta_{j_{k}}=\delta_{1} \quad \text { when } \quad t_{k} \in \mathcal{T}_{\delta_{1}} \quad \text { and } \quad \delta_{j_{k}}=\delta_{2} \quad \text { when } \quad t_{k} \in \mathcal{T}_{\delta_{2}} .
$$

As in Section 2, we introduce a set $\mathfrak{N}_{\delta}^{\mathcal{T}_{\delta}}$ of all $\mathcal{T}_{\delta}$-guided $\mathcal{T}_{\delta}$-proper periodic orbits in $I_{T}$. The following assertion makes it possible to reformulate hypothesis (6) in Theorem 3 in a coordinate form.

Lemma 5. The $\omega$-image of any $(\mathcal{T}$-guided, $\mathcal{T}$-proper $)$ orbit in $\mathcal{O}$ is a $\left(\mathcal{T}_{\delta}\right.$-guided, $\mathcal{T}_{\delta}$-proper $)$ orbit in $\mathcal{O}_{\delta}$ and conversely, any $\left(\mathcal{T}_{\delta}\right.$-guided, $\mathcal{T}_{\delta}$-proper $)$ orbit in $\mathcal{O}_{\delta}$ is an $\omega$-image of a $(\mathcal{T}$-guided, $\mathcal{T}$-proper $)$ orbit in $\mathcal{O}$. In particular, the sets $\mathfrak{N}_{\zeta}^{\mathcal{T}}$ and $\mathfrak{N}_{\delta}^{\mathcal{T}_{\delta}}$ can be empty only simultaneously.

Proof. Let $\zeta_{j}(q)=\hat{q}$ for some $j$. In other words, the sequence $(q, \hat{q})$ is an orbit in $\Gamma$. Then $\omega(\hat{q})=\omega \circ \zeta_{j}(q)=\delta_{j} \circ \omega(q)$, due to (15), and consequently $(\omega(q), \omega(\hat{q}))$ is an orbit in $I_{T}$. If $\delta_{j}(t)=\hat{t}$, then $\omega_{\Gamma}^{-1} \hat{t}=\zeta_{j}\left(\omega_{\Gamma}^{-1}(t)\right)$, so that $(t, \hat{t})$ is the $\omega$-image of an orbit $\left(\omega_{\Gamma}^{-1}(t), \omega_{\Gamma}^{-1}(\hat{t})\right)$ in $\Gamma$. Furthermore, due to (19), for any point $x_{0}=\alpha\left(z_{0}\right)$ the implication

$$
\alpha_{j}^{\prime}\left(z_{0}\right)=0 \Longleftrightarrow \delta_{j}^{\prime}\left(\omega\left(x_{0}\right)\right)=0
$$


holds, and hence, the $\omega$-image of an arbitrary point $x_{0} \in \mathcal{T}_{j}$ belongs to $\mathcal{T}_{\delta_{j}}$ and vice versa. Consequently,

$$
\left(x^{(1)}, \ldots, x^{(n)}\right) \in \mathfrak{N}_{\zeta}^{\mathcal{T}} \Longleftrightarrow\left(\omega\left(x^{(1)}\right), \ldots, \omega\left(x^{(n)}\right)\right) \in \mathfrak{N}_{\delta}^{\mathcal{T}_{\delta}} .
$$

In particular,

$$
\mathfrak{N}_{\zeta}^{\mathcal{T}}=\emptyset \Longleftrightarrow \mathfrak{N}_{\delta}^{\mathcal{T}_{\delta}}=\emptyset .
$$

This completes the proof of the lemma.

Turn now to equation (3). Remembering (see the end of Subsection 2.1) that each function $f \in C_{\langle I\rangle}(D)$ can be represented as $F(\omega(x))$, where $\omega$ solves equation $(4)$, we rewrite this equation in the form

$$
(\mathcal{B F})(z):=\int_{0}^{\alpha_{1}(z)} \int_{0}^{\alpha_{2}(z)} F\left(\omega\left(x_{1}, x_{2}\right)\right) d x_{2} d x_{1}=\tilde{h}(z), \quad z \in I_{Z},
$$

where $\tilde{h}(z)=h(\alpha(z))$. In view of $(10), \mathcal{B} F \in \mathcal{H}\left(I_{Z}\right)$ for all functions $F \in C\left(I_{T}\right)$. It follows that for any function $\tilde{h} \in \mathcal{H}\left(I_{Z}\right)$ equation (23) is equivalent to each of the two equations

$$
(d / d z) \mathcal{B} F=(d / d z) \tilde{h}, \quad z \in I_{Z},
$$

and

$$
\left(d^{2} / d z^{2}\right) \mathcal{B} F=\left(d^{2} / d z^{2}\right) \tilde{h}, \quad z \in I_{Z} .
$$

In detailed notation the first equation has the form

$$
\alpha_{1}^{\prime}(z) \int_{0}^{\alpha_{2}(z)} F\left(\omega\left(\alpha_{1}(z), x_{2}\right)\right) d x_{2}+\alpha_{2}^{\prime}(z) \int_{0}^{\alpha_{1}(z)} F\left(\omega\left(x_{1}, \alpha_{2}(z)\right)\right) d x_{1}=\tilde{h}^{\prime}(z) .
$$

Introduce a function

$$
G(t)=\int_{0}^{t} F(s) d s, \quad t \in I_{T},
$$

and substitute $G^{\prime}$ for $F$ in (26). Integrating by parts reduces the equation obtained to a functional-integral equation

$$
\begin{aligned}
&\left(\alpha_{1}^{\prime} / \omega_{x_{2}} \circ \alpha+\alpha_{2}^{\prime} / \omega_{x_{1}} \circ \alpha\right) G(\omega \circ \alpha)(z)-\left(\alpha_{1}^{\prime} / \omega_{x_{2}}\left(\alpha_{1}, 0\right)\right) G\left(\omega_{1} \circ \alpha\right)(z) \\
&-\quad\left(\alpha_{2}^{\prime} / \omega_{x_{1}}\left(0, \alpha_{2}\right)\right) G\left(\omega_{2} \circ \alpha\right)(z)-\mathcal{K} G(z)=\tilde{h}^{\prime}(z), \quad z \in I_{Z},
\end{aligned}
$$

with the additional condition $G(0)=0$. Here $\mathcal{K}$ is a nonpositive operator from $C\left(I_{Z}\right)$ to $C\left(I_{T}\right)$, that is,

$$
G \geq 0 \Longleftrightarrow \mathcal{K} G \leq 0 .
$$

The latter follows from the explicit form of $\mathcal{K}$,

$$
\begin{aligned}
\mathcal{K} G(z) & =\alpha_{1}^{\prime}(z) \int_{0}^{\alpha_{2}(z)}(G \circ \omega)\left(\alpha_{1}(z), x_{2}\right)\left(1 / \omega_{x_{2}}\left(\alpha_{1}(z), x_{2}\right)\right)_{x_{2}} d x_{2} \\
& +\alpha_{2}^{\prime}(z) \int_{0}^{\alpha_{1}(z)}(G \circ \omega)\left(x_{1}, \alpha_{2}(z)\right)\left(1 / \omega_{x_{1}}\left(x_{1}, \alpha_{1}(z)\right)\right)_{x_{1}} d x_{1}
\end{aligned}
$$


if we take into account inequalities (11), (12) and (8). Substituting $\Omega^{-1}(t)$ for $z$ in (27) and denoting $\tilde{h}\left(\Omega^{-1}(t)\right)$ by $H(t)$ we arrive immediately at the equation

$$
\begin{aligned}
G(t)-\mu_{1}(t) G\left(\delta_{1}(t)\right)-\mu_{2}(t) G\left(\delta_{2}(t)\right) & \\
& =\mathcal{K}_{1} G\left(\Omega^{-1}(t)\right)+H^{\prime}(t), \quad t \in I_{T} .
\end{aligned}
$$

Here $\mathcal{K}_{1}=\left(\alpha_{1}^{\prime} / \omega_{x_{2}}+\alpha_{2}^{\prime} / \omega_{x_{1}}\right)^{-1} \mathcal{K}$ is the nonnegative operator from $C\left(I_{Z}\right)$ to $C\left(I_{T}\right)$ and

$$
\left.\mu_{j}(t)=k_{j}(t) \alpha_{j}^{\prime}\left(\Omega^{-1}\right)(t)\right), \quad j=1,2
$$

where

$$
\left\{\begin{array}{c}
k_{1}(t)=\frac{\omega_{x_{1}} \omega_{x_{2}}\left(\alpha \circ \Omega^{-1}\right)(t)}{\omega_{x_{2}}\left(\alpha_{1}, 0\right)\left(\alpha_{1}^{\prime} \omega_{x_{1}} \circ \alpha+\alpha_{2}^{\prime} \omega_{x_{2}} \circ \alpha\right)\left(\Omega^{-1}\right)(t)}>0, \\
k_{2}(t)=\frac{\omega_{x_{1}} \omega_{x_{2}}\left(\alpha \circ \Omega^{-1}\right)(t)}{\omega_{x_{1}}\left(0, \alpha_{2}\right)\left(\alpha_{1}^{\prime} \omega_{x_{1}} \circ \alpha+\alpha_{2}^{\prime} \omega_{x_{2}} \circ \alpha\right)\left(\Omega^{-1}\right)(t)}<0 .
\end{array}\right.
$$

Remark. If $\boldsymbol{l}$ is a constant vector field, then $\omega$ can be chosen as a linear function. But then $\mathcal{K}_{1}=0$ and (29) becomes a purely functional equation. Much more general functional equations of this type are studied in [4].

From the definition, it follows that

$$
t \in \mathcal{T}_{\delta_{j}} \Longleftrightarrow \mu_{j}(t)=0, \quad j=1,2
$$

4.2. The uniqueness of a solution of equation (3). The required uniqueness is a direct consequence of the following assertion which is interesting all by itself.

Lemma 6 (Maximum principle for a functional-integral equation). If $\mathfrak{N}_{\delta}^{\mathcal{T}_{\delta}}=\emptyset$ and a guiding set $\mathcal{T}_{\delta_{1}}\left(\mathcal{T}_{\delta_{2}}\right.$, respectively) is finite, then any solution $G$ of the homogeneous equation (29) takes its maximum at the point $\gamma_{+}\left(\gamma_{-}\right.$, respectively).

Proof. We assume for definiteness that the set $\mathcal{T}_{\delta_{1}}$ is finite. Take an arbitrary solution $G$ of homogeneous equation (29) and introduce a function

$$
G_{\mathcal{M}}(t)=G(t)-\mathcal{M}, \quad \text { where } \mathcal{M}=\max _{I_{T}} G
$$

Denote

$$
\mathfrak{M}=\left\{t \in I_{T} \mid G(t)=\mathcal{M}\right\} .
$$

As any constant solves the homogeneous equation (29) (which can be verified directly), the same is true with respect to the function $G_{\mathcal{M}}$. If $t_{1}=\gamma_{+}$, then there is nothing to prove. Let $t_{1} \in \mathfrak{M}$ and $t_{1} \neq \gamma_{+}$. Applying equality (29) with $H^{\prime}=0$ to the function $G_{\mathcal{M}}$ and substituting $t_{1}$ for $t$ we arrive at the equality

$$
-\mu_{1}\left(t_{1}\right) G_{\mathcal{M}}\left(\delta_{1}\left(t_{1}\right)\right)-\mu_{2}\left(t_{1}\right) G_{\mathcal{M}}\left(\delta_{2}\left(t_{1}\right)\right)=\mathcal{K}_{1} G_{\mathcal{M}}\left(\Omega^{-1}\left(t_{1}\right)\right) .
$$

In view of (9), (11), (30) and (31), the inequalities

$$
\mu_{1} \geq 0, \quad \mu_{2} \geq 0
$$

hold. As $G_{\mathcal{M}} \leq 0$ in $I_{T}$, the right-hand side in (33) is nonpositive, whereas the left-hand side is nonnegative. Consequently,

$$
\mu_{1}\left(t_{1}\right) G_{\mathcal{M}}\left(\delta_{1}\left(t_{1}\right)\right)+\mu_{2}\left(t_{1}\right) G_{\mathcal{M}}\left(\delta_{2}\left(t_{1}\right)\right)=0 .
$$


If $t_{1} \notin \mathcal{T}_{\delta}$, then, in view of (32),

$$
G_{\mathcal{M}}\left(\delta_{1}\left(t_{1}\right)\right)=G_{\mathcal{M}}\left(\delta_{2}\left(t_{1}\right)\right)=0,
$$

and, hence,

$$
\delta_{1}\left(t_{1}\right) \in \mathfrak{M} \quad \text { and } \quad \delta_{2}\left(t_{1}\right) \in \mathfrak{M} .
$$

But if $t_{1} \in \mathcal{T}_{\delta_{1}}\left(t_{1} \in \mathcal{T}_{\delta_{2}}\right.$, respectively), then, by virtue of (18),

$$
\delta_{1}\left(t_{1}\right) \in \mathfrak{M} \quad\left(\delta_{2}\left(t_{1}\right) \in \mathfrak{M},\right. \text { respectively). }
$$

Comparing these observations with the definition of a $\mathcal{T}_{\delta}$-proper orbit (see the paragraph preceding Lemma 5) we conclude that, along with every point $t_{1} \in \mathfrak{M}$, the next point $t_{2}$ of any $\mathcal{T}_{\delta}$-proper orbit $\left(t_{1}, t_{2}\right)$ also belongs to $\mathfrak{M}$. Since $\delta_{1}$ and $\delta_{2}$ are maps in $I$, this argument can be applied to the point $t_{2}$.

As $\delta_{1}$ and $\delta_{2}$ are maps in $I_{T}$, we can apply this argument to the points $t_{2}=$ $\delta_{1}\left(t_{1}\right)$ or $t_{2}=\delta_{2}\left(t_{1}\right)$ and obtain one or two points $t_{3}=\delta_{j_{2}}\left(t_{2}\right)=\delta_{j_{2}} \circ \delta_{j_{1}}\left(t_{1}\right)$ also belonging to $\mathfrak{M}$. A new orbit $\mathcal{O}=\left(t_{1}, t_{2}, t_{3}\right)$ is also $\mathcal{T}_{\delta}$-proper. Arguing in the same way, we conclude that along with every point $t_{1} \in \mathfrak{M}$ its every $\mathcal{I}_{\delta}$-proper orbit $\mathcal{O}=\left(t_{1}, t_{2}, \ldots\right)$ lies in $\mathfrak{M}$. To prove Lemma 6 it remains to verify that if $\mathfrak{N}_{\delta}^{\mathcal{T}_{\delta}}=\emptyset$, then, given any point $t_{1} \in I_{T}$, there is a $\mathcal{T}_{\delta}$-proper orbit $\mathcal{O}=\left(t_{1}, t_{2}, \ldots\right)$ which converges to $\gamma_{+}$, i.e. $\lim _{n \rightarrow \infty} t_{n}=\gamma_{+}$.

Remark. If $\mathcal{T}_{\delta_{1}}=\emptyset$, i.e. the curve $\Gamma$ has no points with a tangent line parallel to the $x_{2}$-axis, then one can complete the proof in several words. Indeed, in this situation for any point $t_{1} \in I_{T}$ the orbit $\left(t_{1}, \delta_{1}\left(t_{1}\right), \ldots, \delta_{1}^{n}\left(t_{1}\right), \ldots\right)$ is $\mathcal{T}_{\delta}$-proper. Furthermore, by virtue of inequality (17), the sequence $\delta_{1}^{n}\left(t_{1}\right)$ increases, when $n \rightarrow \infty$. Denote $\lim _{n \rightarrow \infty} \delta_{1}^{n}\left(t_{1}\right)=\nu$. It is clear that $\delta_{1}(\nu)=\nu$. But this means that $\nu=\gamma_{+}$.

The general case is more difficult, as any $\mathcal{T}_{\delta}$-proper orbit $\left(t_{1}, t_{2}, \ldots, t_{n}, \ldots\right)$ containing points $t_{k}$ from both sets $\mathcal{T}_{\delta_{1}}$ and $\mathcal{T}_{\delta_{2}}$ does not increase, by virtue of the same inequality (17).

We begin with several assertions.

Proposition 7. If $t \neq \gamma_{0}$ and $\left(t_{1}, t\right),\left(t_{2}, t\right)$ are two $\mathcal{T}_{\delta}$-proper orbits, then $t_{1}=t_{2}$.

Proof. Assume that $t_{1} \neq t_{2}$ and

$$
t=\delta_{j_{1}}\left(t_{1}\right), \quad t=\delta_{j_{2}}\left(t_{2}\right) .
$$

By virtue of Lemma 4(i), $j_{1}=j_{2}$. Denote a common value of these indices by $j$. As $\delta_{j}^{\prime} \geq 0$, it follows from the equality $\delta_{j}\left(t_{1}\right)=\delta_{j}\left(t_{2}\right)$ that $\delta_{j}^{\prime}(t)=0$ for all values $t, t_{1} \leq t \leq t_{2}$. But then $t_{1} \in \mathcal{T}_{\delta_{j}}$, whence $j_{1} \neq j$, as the orbit $\left(t_{1}, t\right)$ is $\mathcal{T}_{\delta}$-proper.

Remark. From a geometrical point of view the latter proposition asserts that two different $\mathcal{T}_{\delta}$-proper orbits cannot enter at the same point (although such orbits can leave the same point $t$ if $t \notin \mathcal{T}_{\delta}$ ).

Proposition 8. If a cyclic orbit $S$ is a part of a $\mathcal{T}_{\delta}$-proper orbit $\mathcal{O}=\left(t_{1}, t_{2}, \ldots\right)$, then $t_{1} \in S$.

Proof. If $t_{1} \notin S$ we let $t_{q}, q \geq 2$, be the first point in $\mathcal{O}$, belonging to $S$, so that

$$
S=\left(t_{q}, \ldots, t_{q+n}\right), \quad t_{q+n}=t_{q}
$$

But then $t_{q-1} \neq t_{q+n-1}$, and applying Proposition 7 leads to a contradiction.

Let

$$
\lambda=\sup \left\{t \mid t \in \mathcal{T}_{\delta_{1}}\right\} .
$$


Proposition 9. There is an integer $\nu$ such that the inequality

$$
\delta_{1}^{\nu}(t)>\lambda
$$

holds for all $t \in I_{T}$.

Proof. The existence of an integer $\nu$ such that $\delta_{1}^{\nu}(-1)>\lambda$ is a consequence of inequality (17). The required relation follows immediately from Lemma 4(i).

The end of the proof of Lemma 6 . We say that a $\mathcal{T}_{\delta}$-proper orbit $\left(t_{1}, t_{2}, \ldots\right)$ is $\delta_{1}$ oriented if for all $k \geq 1$

$$
t_{k} \notin \mathcal{T}_{\delta_{1}} \Rightarrow t_{k+1}=\delta_{1}\left(t_{k}\right) .
$$

It is obvious that any point $t_{1} \in I_{T}$ uniquely defines a $\delta_{1}$-oriented orbit. We first note that, by virtue of the hypotheses, the number $\lambda$ does not equal $\gamma_{+}$, and hence, inequality (34) holds for all $t$ with a constant $\nu$. If $t_{1}>\lambda$, then the orbit $\mathcal{O}=\left(t_{1}, \delta_{1}\left(t_{1}\right), \ldots, \delta_{1}^{n}\left(t_{1}\right), \ldots\right)$ is $\delta_{1}$-oriented and, as was shown (see Remark), $\lim _{n \rightarrow \infty} \delta_{1}^{n}\left(t_{1}\right)=\gamma_{+}$. Let $t_{1} \leq \lambda$. If a $\delta_{1}$-oriented orbit $\mathcal{O}=\left(t_{1}, \ldots\right)$ does not lead to the point $t_{1}$, then it does not contain any cyclic suborbit by Proposition 8 . In this case as $\mathcal{T}_{\delta_{1}}$ is a finite set, there exists a number $m$ such that all the points $t_{m+1}, t_{m+2}, \ldots$ of the orbit $\mathcal{O}$ lie outside of $\mathcal{T}_{\delta_{1}}$. But then $t_{m+\nu}=\delta_{1}^{\nu}\left(t_{m}\right)>\lambda$, and, as above, $t_{k} \rightarrow \gamma_{+}$when $k \rightarrow \infty$.

Consider a concluding situation: $t_{m}=t_{1}$ for a number $m$. Then the periodic suborbit $S=\left(t_{1}, \ldots, t_{m}\right)$ of $\mathcal{O}$ cannot be $\mathcal{T}_{\delta}$-guided, by virtue of the hypothesis $\mathfrak{N}_{\delta}=\emptyset$. Consequently, $S$ contains a point $t_{q}, 1 \leq q \leq m-1$, which does not belong to $\mathcal{T}_{\delta}$. Then we introduce a new point $\widehat{t}_{q+1}=\delta_{2}\left(t_{q}\right)$ (in contrast to the point $\left.t_{q+1}=\delta_{1}\left(t_{q}\right) \in S\right)$. Consider now a $\delta_{1}$-oriented orbit $\mathcal{O}_{1}=\left(\widehat{t}_{q+1}, \widehat{t}_{q+2}, \ldots\right)$. Being $\mathcal{T}_{\delta}$-proper this orbit has no common points with $S$. Indeed, if it is not true, $\widehat{t}_{p}$ is the first point of this kind, and $\widehat{t}_{p}=t_{r}, 1 \leq r \leq m$, then, by virtue of Proposition $7, \widehat{t}_{p-1}=t_{r-1}$. But the latter is not possible. By Proposition 8 , the $\mathcal{T}_{\delta}$-proper orbit $t_{q}, \widehat{t}_{q+1}, \widehat{t}_{q+2}, \ldots$ does not contain periodic suborbits. Hence, as above, all the points $\widehat{t}_{q+p+m}, m \geq 1$, of the orbit $\mathcal{O}_{1}$ starting with some number $p$ have the form

$$
\widehat{t}_{q+p+m}=\delta_{1}^{m}\left(\widehat{t}_{q+p}\right) .
$$

This means that the orbit $\mathcal{O}_{1}$ converges to $\gamma_{+}$, and so does the sewed orbit

$$
\mathcal{O}=\left(t_{1}, \ldots, t_{q}, \widehat{t}_{q+1}, \ldots, \widehat{t}_{q+p}, \widehat{t}_{q+p+1}, \ldots\right) .
$$

This completes the proof of the maximum principle.

To prove the uniqueness of a solution of equation (23) turn to the homogeneous equation (23) and prove that its solution $F$ is zero. Indeed, by definition, $F^{\prime}(t)=$ $G(t)$ where $G$ is a solution of the homogeneous equation (29). By the maximum principle, we have

$$
\max _{\left[\gamma_{-}, \gamma_{+}\right]} G=G\left(\gamma_{+}\right) \quad\left(\text { or } \max _{\left[\gamma_{-}, \gamma_{+}\right]} G=G\left(\gamma_{-}\right)\right) .
$$

As equation (29) is linear, we conclude, replacing $G$ by $-G$, that

$$
\min _{\left[\gamma_{-}, \gamma_{+}\right]} G=G\left(\gamma_{+}\right) \quad\left(\text { or } \min _{\left[\gamma_{-}, \gamma_{+}\right]} G=G\left(\gamma_{-}\right), \text {respectively }\right) .
$$

Thus, $G \equiv$ const, and hence, $F \equiv 0$. This completes the proof of the uniqueness in Theorem 3(I). 
4.3. The existence of a solution of equation (3). As we know, the equation in question is equivalent to both equations (24) and (25). Having proved the uniqueness of a solution of equation (24) (see Subsection 4.2) we established at the same time the uniqueness theorem for equation (25). Consequently, the required existence follows if we prove that the linear operator $\left(d^{2} / d z^{2}\right) \mathcal{B}$ in the space $C\left(I_{Z}\right)$ is a Fredholm operator, and its index equals zero. It is worth mentioning that the operator $d^{2} / d z^{2}$ is an isomorphism between the spaces $\mathcal{H}\left(I_{Z}\right)=\left(C^{2} \cap C_{0}\right)\left(I_{Z}\right)$ and $C\left(I_{Z}\right)$. To represent equation (25) in an expanded form we differentiate equation (26). Denoting $\tilde{h}^{\prime \prime}(z)$ by $\tilde{H}(z)$ we arrive after some identical transformations at the functional-integral equation

$$
\begin{aligned}
& {\left[\alpha_{1}^{\prime 2}(z) \frac{\omega_{x_{1}}(\alpha(z))}{\omega_{x_{2}}(\alpha(z))}+\alpha_{2}^{\prime 2}(z) \frac{\omega_{x_{2}}(\alpha(z))}{\omega_{x_{1}}(\alpha(z))}+2 \alpha_{1}^{\prime} \alpha_{2}^{\prime}(z)\right] F(\omega(\alpha(z)))} \\
& \quad-\alpha_{1}^{\prime 2}(z) \frac{\omega_{x_{1}}}{\omega_{x_{2}}}\left(\alpha_{1}(z), 0\right) F\left(\omega\left(\alpha_{1}(z), 0\right)\right)-\alpha_{2}^{\prime 2}(z) \frac{\omega_{x_{2}}}{\omega_{x_{1}}}\left(0, \alpha_{2}(z)\right) F\left(\omega\left(0, \alpha_{2}(z)\right)\right) \\
& =\alpha_{1}^{\prime 2}(z) \int_{0}^{\alpha_{2}(z)} F\left(\omega\left(\alpha_{1}(z), x_{2}\right)\right)\left(\frac{\omega_{x_{1}}}{\omega_{x_{2}}}\left(\alpha_{1}(z), x_{2}\right)\right)_{x_{2}} d x_{2} \\
& \quad+\alpha_{2}^{\prime 2}(z) \int_{0}^{\alpha_{1}(z)} F\left(\omega\left(x_{1}, \alpha_{2}(z)\right)\right)\left(\frac{\omega_{x_{2}}}{\omega_{x_{1}}}\left(x_{1}, \alpha_{2}(z)\right)\right)_{x_{1}} d x_{1}+\tilde{H}(z) .
\end{aligned}
$$

Substituting $\Omega^{-1}(t)$ for $z$ yields

$$
F(t)-\rho_{1}(t) F\left(\delta_{1}(t)\right)-\rho_{2}(t) F\left(\delta_{2}(t)\right)=\mathcal{N} F(t)+H(t), \quad t \in I_{T},
$$

where $\mathcal{N}$ is the integral operator

$$
\begin{aligned}
\mathcal{N}: F & \mapsto \alpha_{1}^{\prime^{2}}(t) \int_{0}^{\alpha_{2}(t)} F\left(\omega\left(\alpha_{1}(t), x_{2}\right)\right)\left(\frac{\omega_{x_{1}}}{\omega_{x_{2}}}\left(\alpha_{1}(t), x_{2}\right)\right)_{x_{2}} d x_{2} \\
& +\alpha_{2}^{\prime 2}(t) \int_{0}^{\alpha_{1}(t)} F\left(\omega\left(x_{1}, \alpha_{2}(t)\right)\right)\left(\frac{\omega_{x_{2}}}{\omega_{x_{1}}}\left(x_{1}, \alpha_{2}(t)\right)\right)_{x_{1}} d x_{1}+\tilde{H}(t),
\end{aligned}
$$

$H(t)$ stands for the function $\tilde{H}\left(\Omega^{-1}(t)\right)$ and

$$
\begin{aligned}
& \rho_{1}(t)=\left.\frac{\alpha_{1}^{2}(z) \lambda\left(\alpha_{1}(z), 0\right)}{\alpha_{1}^{\prime 2}(z) \lambda(\alpha(z))+\alpha_{2}^{\prime 2}(z)(1 / \lambda)(\alpha(z))-2 \alpha_{1}^{\prime} \alpha_{2}^{\prime}(z)}\right|_{z=\Omega^{-1}(t)}, \\
& \rho_{2}(t)=\left.\frac{\alpha_{2}^{\prime 2}(z)(1 / \lambda)\left(0, \alpha_{2}(z)\right)}{\alpha_{1}^{\prime 2}(z) \lambda(\alpha(z))+\alpha_{2}^{\prime 2}(z)(1 / \lambda)(\alpha(z))-2 \alpha_{1}^{\prime} \alpha_{2}^{\prime}(z)}\right|_{z=\Omega^{-1}(t)}
\end{aligned}
$$

with $\lambda(x)=\left(\lambda_{2} / \lambda_{1}\right)(x)($ see $(4))$.

Remark. If the vector field $\boldsymbol{l}$ is proportional to a constant one, then $\mathcal{N}=0$ and (35) becomes the purely functional equation

$$
F(t)-\rho_{1}(t) F\left(\delta_{1}(t)\right)-\rho_{2}(t) F\left(\delta_{2}(t)\right)=H(t)
$$

with respect to an unknown function $F \in C\left(I_{T}\right)$.

The integral operator $\mathcal{N}$, as follows from the classical Arzela criterion, is a compact operator in $C\left(I_{T}\right)$. As to the coefficients $\rho_{1}(t)$ and $\rho_{2}(t)$, what is important 
are the following properties:

(i) $\rho_{1}(t) \rho_{2}(t) \geq 0, \quad t \in I_{T}$;

(ii) $0<\rho_{1}(t)+\rho_{2}(t) \leq 1$ for all $t \in I_{T}$ and $\rho_{1}(t)+\rho_{2}(t)<1$ for $t \notin \mathcal{T}_{\delta}$

(iii) $\rho_{j^{\prime}}^{\prime}(t)=0 \Longleftrightarrow t \in \mathcal{T}_{\delta_{j}}, \quad j \neq j^{\prime}$.

The first inequality in (ii) follows from (9), (11) and (7), and the second is based on (7). The implication (iii) is a consequence of (22).

Introduce a linear operator $L$ in $C\left(I_{T}\right)$ :

$$
L: F \mapsto \rho_{1} F \circ \delta_{1}+\rho_{2} F \circ \delta_{2} .
$$

In view of (ii), the norm of this operator $\|L\|$ does not exceed 1.3 If $\|L\|<1$, then the operator $E-L$ on the left-hand side of (35) (with $E$ the identical operator in $C\left(I_{T}\right)$ ) is invertible. In this case the required Fredholm property as well as the equality ind $\left(d^{2} / d z^{2}\right) \mathcal{B}=0$ follows from the well-known

Proposition 10. If $R$ is an invertible operator in a Banach space $B$ and $\mathcal{N}$ is a compact operator in $B$, then $R-\mathcal{N}$ is a Fredholm operator in $B$, and $\operatorname{ind}(R-\mathcal{N})=0$.

The following proposition relating to the case $\|L\| \geq 1$ is also well known.

Proposition 11. If $L$ is a linear operator in $B$ and $\left\|L^{m}\right\|<1$ for some integer $m$, then the operator $E-L$ is invertible.

For completeness both propositions are proved in the Appendix.

From what was said above it follows that the required existence of a solution to equation (35) (and consequently, to equation (3)) is a direct consequence of the following assertion.

Lemma 12. If $\mathfrak{N}_{\delta}^{\mathcal{T}_{\delta}}=\emptyset$ and at least one of the guiding sets $\mathcal{T}_{\delta_{1}}$ and $\mathcal{T}_{\delta_{2}}$ is finite, then there is an integer $m$ such that $\left\|L^{m}\right\|<1$.

Proof. We note that for an arbitrary integer $N>0$ the function $L^{N} f$ can be represented in the form

$$
\left(L^{N} f\right)(t)=\sum_{j_{1}, \ldots, j_{N}=1}^{2} \rho_{j_{1}}(t) \rho_{j_{2}}\left(\delta_{j_{1}}(t)\right) \cdots \rho_{j_{N}}\left(\delta_{j_{N-1}} \circ \cdots \circ \delta_{j_{1}}(t)\right) f\left(\delta_{J}(t)\right)
$$

where $J=\left(j_{1}, j_{2}, \ldots, j_{N}\right)$. Indeed, for $N=1$ this is true. To apply the induction, assume this equality to be valid for some $N$ and prove that it is valid for $N+1$. But this is evident, since, by the definition of $L$ and in view of (37), we have

$$
\begin{aligned}
& L^{N+1} f(t)=L\left(L^{N} f\right)=\sum_{j_{0}=1}^{2} \rho_{j_{0}}(t)\left(L^{N} f\right)\left(\delta_{j_{0}}(t)\right) \\
& \quad=\sum_{j_{0}, j_{1}, \ldots, j_{N}=1}^{2} \rho_{j_{0}}(t) \rho_{j_{1}}\left(\delta_{j_{0}}(t)\right) \cdots \rho_{j_{N}}\left(\delta_{j_{N-1}} \circ \cdots \circ \delta_{j_{0}}(t)\right) f\left(\delta_{J}(t)\right)
\end{aligned}
$$

with $J=\left(j_{0}, j_{1}, \ldots, j_{N}\right)$.

\footnotetext{
${ }^{3}$ We consider a standard norm $\|\cdot\|$ in $C\left(I_{T}\right):\|F\|=\max _{t \in I_{T}}|F(t)|$.
} 
Applying the triangle inequality we conclude, with the help of (37), that for an arbitrary function $f \in C\left(I_{T}\right)$ and at any point $t \in I_{T}$ the inequality

$$
\left|L^{N} f(t)\right| \leq \sum_{j_{1}, \ldots, j_{N}=1}^{2} \rho_{j_{1}}(t) \rho_{j_{2}}\left(\delta_{j_{1}}(t)\right) \cdots \rho_{j_{N}}\left(\delta_{j_{N-1}} \circ \cdots \delta_{j_{1}}(t)\right)\|f\|
$$

holds. Let us prove that for an arbitrary point $t \in I_{T}$ one can find an integer $N$ and a number $\gamma<1$ such that for all functions $f$ with $\|f\|=1$ the inequality

$$
\left|L^{N} f(t)\right|<\gamma
$$

holds. If $t \notin \mathcal{T}_{\delta}$, then $\rho_{j_{1}}(t)+\rho_{j_{2}}(t)<1$, in view of property (ii), and hence, inequality (38) follows with $N=1$. Let $t \in \mathcal{T}_{\delta}$. In proving the maximum principle we established that under the hypotheses of the lemma there is a multi-index $\widehat{J}=$ $\left(j_{1}, \ldots, j_{N-1}\right), N \geq 2$, such that the corresponding orbit $\mathcal{O}=\left(t, \delta_{j_{1}}(t), \ldots, \delta_{\widehat{J}}(t)\right)$ is $\mathcal{T}_{\delta}$-proper and $\delta_{\widehat{J}}(t) \notin \mathcal{T}_{\delta}$. Let $N$ be the minimal integer satisfying this condition. Then, by the definition of $\mathcal{T}_{\delta}$-proper orbit, we have

$$
\rho_{j_{1}^{\prime}}(t)=0, \rho_{j_{2}^{\prime}}\left(\delta_{j_{1}}(t)\right)=0, \ldots, \rho_{j_{N-1}^{\prime}}\left(\delta_{j_{N-2}} \circ \cdots \circ \delta_{j_{1}}(t)\right)=0,
$$

with $j_{k}^{\prime} \neq j_{k}$ for all $k$, and hence, in view of (37),

$$
\left|L^{N} f(t)\right| \leq \rho_{j_{1}}(t) \rho_{j_{2}}\left(\delta_{j_{1}}(t)\right) \cdots \rho_{j_{N-1}}\left(\delta_{j_{N-2}} \circ \cdots \circ \delta_{j_{1}}(t)\right)\left(\rho_{1}\left(\delta_{\widehat{J}}(t)\right)+\rho_{2}\left(\delta_{\widehat{J}}(t)\right)\right) .
$$

By virtue of property (ii), the first $N-1$ factors on the right-hand side are not greater than 1 , whereas the latter one is strictly less than 1 . This completes the proof of inequality (38).

By continuity, this inequality remains valid at all points of some neighborhood $\mathcal{U}$ of the point $t$ for the same number $N$, probably, with a larger constant $\gamma<1$. Let $\left\{\mathcal{U}_{j}\right\}_{j=1}^{k}$ be a finite subsystem of these neighborhoods, and let $N_{j}$ and $\gamma_{j}$ be the corresponding constants. Setting $m=\max N_{j}$ and $\gamma=\max \gamma_{j}$ we arrive at the desired inequality $\left\|L^{m}\right\|<1$. This completes the proof of Lemma 12, and hence, the proof of part (I) in Theorem 3.

4.4. Proof of part (II). In proving part (II) we follow completely the proof of part (I), including the notation being used. To begin with we establish the uniqueness of solution $F$ to equation (23). Note that the function $\omega\left(x_{1}, x_{2}\right)$ in equation (23) can be chosen now as linear, since $\boldsymbol{l}$ is a constant vector field. But then both functions $\omega_{x_{1}}$ and $\omega_{x_{2}}$ are constants, and combining these observations with (30) and (31) leads to the relations

$$
\mu_{1}(t)+\mu_{2}(t)=1, \quad t \in I_{T}
$$

and $\mathcal{K}_{1}=0$. The homogeneous equation (29), thus, becomes

$$
G(t)-\mu_{1}(t) G\left(\delta_{1}(t)\right)-\mu_{2}(t) G\left(\delta_{2}(t)\right)=0, \quad t \in I_{T},
$$

and the required uniqueness follows, if we establish that $G(t) \equiv$ const. It can be verified directly that if both sets $\mathcal{T}_{1}$ and $\mathcal{T}_{2}$ are infinite, and the geometric hypotheses in Theorem 1, related to the sets $\mathcal{T}_{1}^{\prime}$ and $\mathcal{T}_{2}^{\prime}$, are fulfilled, then the inequality

$$
\min \left\{t \mid t \in \mathcal{T}_{\delta_{1}}^{\prime}\right\}>\max \left\{t \mid t \in \mathcal{T}_{\delta_{2}}^{\prime}\right\}
$$

holds. By Theorem 2 in [4], if $\mathfrak{N}_{\delta}^{\mathcal{T}_{\delta}}=\emptyset$, then under hypotheses (39) and (41) any solution $G$ of equation (40) takes its maximum and minimum at boundary points $\gamma_{-}$and $\gamma_{+}$of the interval $I_{T}$. 
Let us substitute consecutively $\gamma_{-}$and $\gamma_{+}$for $t$ in (40). As $\gamma_{-}$and $\gamma_{+}$are fixed points of the maps $\delta_{1}$ and $\delta_{2}$, respectively (Lemma 4), we arrive, in view of (39), at relations

$$
\mu_{1}\left(\gamma_{-}\right)\left(G\left(\gamma_{-}\right)-G(0)\right)=\mu_{2}\left(\gamma_{+}\right)\left(G\left(\gamma_{+}\right)-G(0)\right)=0 .
$$

Note that $\mu_{1}\left(\gamma_{-}\right) \mu_{2}\left(\gamma_{+}\right) \neq 0$, by virtue of the transversality $\Gamma$ to the vector fields $\boldsymbol{l}_{1}$ and $\boldsymbol{l}_{2}$ at the boundary points of $\Gamma$ (see Subsection 2.1). Therefore, $G\left(\gamma_{-}\right)=G\left(\gamma_{+}\right)$, and hence, by the above, $\max G=\min G$. Thus, $G \equiv$ const.

In proving the existence of a solution to equation (3) we repeat word for word the arguments at the beginning of Subsection 4.3, and using the Remark in Subsection 4.3 , arrive at equation (36). It remains to prove the following analog of Lemma 12.

Lemma 13. Assume that the semigroup $\Phi_{\delta}$ is generated by the maps $\delta_{1}$ and $\delta_{2}$ in $I_{T}$, described in Lemma 4 , that the sets $\mathcal{T}_{\delta_{1}}^{\prime}$ and $\mathcal{T}_{\delta_{2}}^{\prime}$ satisfy hypothesis (41), if none of them is finite, and that $\mathcal{T}_{\delta}{ }^{\prime} \cap \partial I_{T}=\emptyset$. Then $\left\|L^{m}\right\|<1$ for some integer $m$, provided that $\mathfrak{N}_{\delta}^{\mathcal{T}_{\delta}}=\emptyset$.

The proof of this lemma coincides with the proof of Lemma 12 with the only exception: the reference to the maximum principle should be replaced by the reference to Theorem 1 in [5]. The corresponding assertion in [5] looks as follows: Under the hypotheses of Lemma 13, for an arbitrary point $t_{1} \in I_{T}$ there is a $\mathcal{T}_{\delta}$-proper orbit $\mathcal{O}=\left(t_{1}, t_{2}, \ldots\right)$ converging to the boundary of $I_{T}$ if and only if $\mathfrak{N}_{\delta}^{\mathcal{T}_{\delta}}=\emptyset$. This completes the proof of the "if" part in Theorem 3(II).

To prove the necessity of hypothesis (6) assume that

$$
\mathfrak{N}_{\zeta}^{\mathcal{T}} \neq \emptyset .
$$

Let $\mathcal{O}_{q}=\left(q_{1}, q_{2}, \ldots, q_{n+1}\right)$ be a $\mathcal{T}$-guided $\mathcal{T}$-proper periodic orbit in $\Gamma$ with $J=$ $\left(j_{1}, j_{2}, \ldots, j_{n}\right)$. According to Lemma 5 , there is a $\mathcal{T}_{\delta}$-guided $\mathcal{T}_{\delta}$-proper periodic orbit $\mathcal{O}=\left(t_{1}, t_{2}, \ldots, t_{n+1}\right)$ in $I_{T}$ responding to the same multi-index $J$. The latter means, according to definitions (see Subsection 4.1), that

$$
\left\{\begin{array}{l}
\text { (i) } t_{1}=t_{n+1} ; \\
\text { (ii) } \quad t_{k+1}=\delta_{j_{k}}\left(t_{k}\right), 1 \leq k \leq n, \\
\text { (iii) } \quad \delta_{j_{k}^{\prime}}^{\prime}\left(t_{k}\right)=0,1 \leq k \leq n,
\end{array}\right.
$$

where $j_{k}^{\prime} \neq j_{k}$. Return to equation (29). As was mentioned above, now it takes the form

$$
G(t)-\mu_{1}(t) G\left(\delta_{1}(t)\right)-\mu_{2}(t) G\left(\delta_{2}(t)\right)=H^{\prime}(t) .
$$

Let us substitute $t_{1}$ for $t$ in (43). Using consecutively relations (42) and (39) we conclude that one of the numbers $\delta_{j}^{\prime}\left(t_{1}\right), j=1,2$, equals zero, which means, in view of $(19)$, that $\mu_{j}\left(t_{1}\right)=0$, whereas $\mu_{j^{\prime}}\left(t_{1}\right)=1$ and $\delta_{j^{\prime}}\left(t_{1}\right)=t_{2}$ for $j^{\prime} \neq j$. This results in the relation

$$
G\left(t_{1}\right)-G\left(t_{2}\right)=H^{\prime}\left(t_{1}\right)
$$


Using this procedure consecutively for $t=t_{2}, \ldots, t=t_{n}$ and applying the periodicity of the orbit in question on the last step, we arrive at the system of relations

$$
\begin{aligned}
G\left(t_{1}\right)-G\left(t_{2}\right) & =H^{\prime}\left(t_{1}\right), \\
G\left(t_{2}\right)-G\left(t_{3}\right) & =H^{\prime}\left(t_{2}\right), \\
\ldots & \\
G\left(t_{n}\right)-G\left(t_{1}\right) & =H^{\prime}\left(t_{n}\right) .
\end{aligned}
$$

Adding all of them together we get

$$
\sum_{j=1}^{n} H^{\prime}\left(t_{j}\right)=0
$$

By the definition of $H^{\prime}$, this is equivalent to the relation

$$
\sum_{j=1}^{n} \partial_{s} h\left(q_{j}\right)=0
$$

Here $\partial_{s}$ is the differentiation with respect to the natural parameter on $\Gamma$. Thus, each $\mathcal{T}$-guided $\mathcal{T}$-proper cycle $\mathcal{O}_{q}=\left(q_{1}, \ldots, q_{n+1}\right)$ generates a relation (44) involving any given function $h$ in (3). A violation of this relation leads to the unsolvability of equation (3) with this $h$. In other words, each orbit from $\mathfrak{N}_{\zeta}^{\mathcal{T}}$ represents an obstruction when constructing the inverse operator $\mathcal{B}^{-1}$. Thus, we have proved the "only if" part in Theorem 3(II). This completes the proof of Theorem 3.

Remark. Note that the problem of the completeness of a system of the above obstruction as well as the problem of whether or not these conditions are sufficient for the solvability of problem (3) still remain open.

\section{Proof of Theorem 1}

Let $Y: \widetilde{D} \rightarrow D$ be the diffeomorphism described in Subsection 3.1 and let $\widetilde{\Gamma}=Y(\Gamma)$. Introduce the vector field $\mathbf{L}=\lambda_{1} \mathbf{e}_{1}+\lambda_{2} \mathbf{e}_{2}$ in $\widetilde{D}$. As was shown in Section 3, equation (3) we are dealing with has the form

$$
\int_{D_{q}} F(\omega(y)) d \sigma=h(q), \quad q \in \Gamma,
$$

where $\omega(y)$ is a nonsingular solution of the differential equation

$$
(\partial / \partial \mathbf{I}) \omega(y)=0, \quad y \in B,
$$

and $F$ is an unknown continuous function on the range of $\omega$. It is obvious that the change of variable

$$
y=Y(x), \quad x \in \widetilde{D},
$$

reduces equation (45) in an equivalent manner to the equation

$$
\int_{\widetilde{D}_{\widetilde{q}}} F(\Omega(x)) d x_{1} d x_{2}=h(\widetilde{q}), \quad \widetilde{q} \in \widetilde{\Gamma},
$$

where $\Omega(x)=\lambda_{2} x_{1}-\lambda_{1} x_{2}$ is a solution of the differential equation with constant coefficients

$$
(\partial / \partial \mathbf{L}) \Omega(x)=0, \quad x \in \widetilde{D} .
$$


As was mentioned in Subsection 3.1, the triangle $\widetilde{D}$ and the vector fields $\boldsymbol{e}_{1}$ and $\mathbf{e}_{2}$ satisfy the hypotheses $1^{\circ}$ and $2^{\circ}$ in Subsection 2.1. This makes it possible (analogously to Section 2) to introduce the maps $\widetilde{\zeta}_{1}$ and $\widetilde{\zeta}_{2}$ in $\widetilde{\Gamma}$ along with the semigroup $\Phi_{\widetilde{\zeta}}$, the guiding sets $\widetilde{\mathcal{T}}_{1}, \widetilde{\mathcal{T}}_{2}$ and $\widetilde{\mathcal{T}}=\widetilde{\mathcal{T}}_{1} \cup \widetilde{\mathcal{T}}_{2}$, and to define the corresponding $\tilde{\mathcal{T}}$ guided, $\widetilde{\mathcal{T}}$-proper orbits related to $\Phi_{\widetilde{\zeta}}$. Denote by $\mathfrak{N}_{\widetilde{\zeta}}^{\widetilde{\mathcal{T}}}$ the set of all $\widetilde{\mathcal{T}}$-guided, $\widetilde{\mathcal{T}}$-proper periodic orbits. With the help of the obvious relation

$$
y \circ \widetilde{\zeta}_{j}=\zeta_{j} \circ y \quad \text { on } \widetilde{\Gamma}, j=1,2,
$$

it can be verified directly that:

$\alpha)$ the pairs of the guiding sets $\left(\mathcal{T}_{1}, \mathcal{T}_{2}\right)$ and $\left(\widetilde{\mathcal{T}}_{1}, \widetilde{\mathcal{T}}_{2}\right)$ satisfy the geometric hypothesis in Theorem 1 only simultaneously;

$\beta$ ) the relations

$$
\mathfrak{N}_{\zeta}^{\mathcal{T}}=\emptyset \quad \text { and } \quad \mathfrak{N}_{\widetilde{\zeta}}^{\widetilde{\mathcal{T}}}=\emptyset
$$

are equivalent.

As a consequence, we conclude that equation (45) satisfies all the conditions of Theorem 3(II), and, therefore, is uniquely solvable in the space $C_{\langle\mathbf{L}\rangle}(\widetilde{D})$ for all functions $h \in \mathcal{H}(\widetilde{\Gamma})$ if and only if $\mathfrak{N}_{\widetilde{\zeta}}^{\widetilde{\mathcal{T}}}=\emptyset$. In view of the above-mentioned equivalence of problems (45) and (46), the same is true with respect to problem (45), the spaces $C_{\langle 1\rangle}(D)$ and $\mathcal{H}(\Gamma)$ and the condition $\mathfrak{N}_{\zeta}^{\mathcal{T}}=\emptyset$.

To complete the proof of Theorem 1 it remains to show that the inverse operator $\mathcal{B}^{-1}$ (whose existence follows from the uniqueness of a solution to equation (3)) is bounded: $\mathcal{H}(\Gamma) \rightarrow C_{\langle I\rangle}(D)$. But we have proved that this operator is defined on the whole Banach space $\mathcal{H}(\Gamma)$ (the existence part in Theorem 3). In order to use the Banach closed graph theoren 4 and thus to establish the desirable boundedness, we have only to show that the operator $\mathcal{B}: C_{\langle I\rangle}(D) \rightarrow \mathcal{H}(\Gamma)$ is bounded. The latter is equivalent to the estimate

$$
\|\mathcal{B} F, \mathcal{H}(\Gamma)\| \leq c\|F, C(\bar{D})\|, \quad F \in C_{\langle I\rangle}(\bar{D}),
$$

with $c>0$ a constant. But

$$
\|\mathcal{B} F, \mathcal{H}(\Gamma)\|=\max _{I_{Z}}\left|\left(d^{2} / d z^{2}\right) \mathcal{B} F\right|+\max _{I_{Z}}|\mathcal{B} F|,
$$

and making use of the explicit form of the operator $\left(d^{2} / d z^{2}\right) \mathcal{B}$ (see the relation preceding (35)) we arrive easily at the latter inequality (without special assumptions about the coefficients $\lambda_{1}$ and $\lambda_{2}$ ). This completes the proof of Theorem 1 .

\section{Proof of Theorem 2}

Just as in proving Theorem 1 we reduce equation (45) to the equivalent equation (46). The corresponding equation (47), as can be verified directly, takes the form

$$
\left(\lambda_{1}(x) \partial / \partial x_{1}+\lambda_{2}(x) \partial / \partial x_{2}\right) \Omega=0,
$$

\footnotetext{
${ }^{4}$ This theorem states: if $B_{1}$ and $B_{2}$ are Banach spaces and $L: B_{1} \rightarrow B_{2}$ is a continuous one-to-one linear operator on $B_{1}$ onto $B_{2}$, then its inverse $L^{-1}$ is also continuous.
} 
where the coefficients $\lambda_{1}$ and $\lambda_{2}$, in view of the restrictions (i) and (ii), satisfy the conditions

$$
\begin{aligned}
& \lambda_{1}=\lambda_{1}\left(x_{2}\right) \quad \text { with } \quad \lambda_{1}^{\prime}\left(x_{2}\right) \leq 0, \\
& \lambda_{2}=\lambda_{2}\left(x_{1}\right) \quad \text { with } \quad \lambda_{2}^{\prime}\left(x_{1}\right) \leq 0,
\end{aligned}
$$

and, therefore, conditions (7).

It follows that the function

$$
\Omega(x)=\int_{0}^{x_{2}} \lambda_{1}(s) d s-\int_{0}^{x_{1}} \lambda_{2}(s) d s
$$

not only solves equation (48), but also satisfies condition (8). By Theorem 3(I), equation (46) possesses the required solvability properties under the hypothesis

$\mathfrak{N}_{\widetilde{\mathcal{L}}}^{\widetilde{\mathcal{T}}}=\emptyset$. In view of the above-mentioned equivalence of the two integral equations, the same remains true with respect to equation (45) and relation $\mathfrak{N}_{\zeta}^{\mathcal{T}}=\emptyset$. This completes the proof of Theorem 2.

\section{FIRST BOUNDARY PROBLEM FOR HYPERBOLIC DIFFERENTIAL EQUATIONS}

As an application of the obtained results a new boundary problem for a wide class of hyperbolic differential operators in the plane will be studied in this section. The main distinctive features of this problem are that it is considered in a bounded domain, and the value of an unknown function is given on the whole boundary of the domain. In this connection it is worth mentioning that, in the framework of the classical theory of PDE, boundary problems for hyperbolic equations are usually considered in domains which are intimately connected with the corresponding equation (half-space, half-cylinder, an angle between characteristics in $\mathbb{R}^{2}$, etc). If the domain is bounded, a part of the boundary is usually free of a priori information about unknown solutions. The evolutionary character of hyperbolic equations seems to impose a taboo on a priori information about a solution on the whole boundary of a bounded domain. However, as we show below (see Theorem 14), for a wide class of hyperbolic equations this taboo can be lifted. In domains closely connected with the corresponding hyperbolic differential operators solutions of equations in question are uniquely defined if their values on the boundaries of these domains are given (the first boundary problem).

7.1. Statement of the problem. For the sake of brevity we restrict ourselves to a homogeneous differential operator with constant coefficients.

In the $(x, y)$-plane $\mathbb{R}^{2}$ we consider an arbitrary homogeneous $x$-strictly hyperbolic operator $P\left(\partial_{x}, \partial_{y}\right)$ of 3 rd order. The $x$-strictly hyperbolicity means that the characteristic polynomial $P(\tau, \lambda)$ has, for any $\lambda \neq 0$, three distinct real roots in $\tau$. It follows that the operator $P=P\left(\partial_{x}, \partial_{y}\right)$ can be uniquely represented in the form

$$
P\left(\partial_{x}, \partial_{y}\right)=a\left(\partial_{x}-a_{1} \partial_{y}\right)\left(\partial_{x}-a_{2} \partial_{y}\right)\left(\partial_{x}-a_{3} \partial_{y}\right)
$$

with some constants $a, a_{1}, a_{2}, a_{3}$, where $a_{j} \neq a_{k}$ for $j \neq k$. The characteristics of the operator $P$ are straight lines

$$
y+a_{1} x=\text { const }, \quad y+a_{2} x=\text { const }, \quad y+a_{3} x=\text { const. }
$$

Let $\boldsymbol{l}_{1}, \boldsymbol{l}_{2}$ and $\boldsymbol{l}_{3}$ be the unique vector fields in $\mathbb{R}^{2}$ parallel to these lines, respectively. Denote by $\mathcal{R}_{1}, \mathcal{R}_{2}, \ldots, \mathcal{R}_{6}$ characteristic rays beginning at some point 0 . 
Choose any triple of neighboring rays $\mathcal{R}_{j}$, say, $\mathcal{R}_{1}, \mathcal{R}_{2}$ and $\mathcal{R}_{3}$. Let $\mathcal{R}_{3}$ be the ray lying between $\mathcal{R}_{1}$ and $\mathcal{R}_{2}$. Consider a curvilinear triangle $D=O A_{1} A_{2}$ with sides $O A_{1} \subset \mathcal{R}_{1}, O A_{2} \subset \mathcal{R}_{2}$. As to the side $\Gamma=A_{1} A_{2}$, it is assumed to be an arbitrary smooth curve without singularities which is transversal to $O A_{1}$ and $O A_{2}$ (cf. Subsection 2.1). We suppose the closure $\bar{D}$ satisfies hypotheses $1^{\circ}$ and $2^{\circ}$ in Subsection 2.1. It follows, in particular, that $\Gamma$ is transversal to the vector field $\boldsymbol{l}_{3}$.

We now formulate the first boundary problem for the above operator $P\left(\partial_{x}, \partial_{y}\right)$ and the domain $D$ :

given functions $F \in C(\bar{D})$ and $h \in C(\partial D)$ find a solution $u$ of the problem

$$
P u=F \quad \text { in } D, \quad u=h \text { on } \partial D \text {. }
$$

We call a function $u$ in $\bar{D}$ a generalized solution of problem (50) if $u \in C^{2}(D), u=h$ on $\partial D$, and for all functions $\varphi \in C_{0}^{\infty}(D)$

$$
\int_{\mathbb{R}^{2}} u^{t} P \varphi d x d y=\int_{\mathbb{R}^{2}} F \varphi d x d y,
$$

where ${ }^{t} P$ is the formally adjoint differential operator.

7.2. The formulation of the result and a sketch of the proof. To formulate the main result related to the solvability of problem (45) let us consider the semigroup $\Phi_{\zeta}$ of maps in $\Gamma$ introduced in Subsection 2.2 with $\boldsymbol{l}=\boldsymbol{l}_{3}$. The guiding sets $\mathcal{T}_{j}$ considered in Subsection 2.2 are now nothing but the sets of characteristic points in $\Gamma$ with respect to the operator $P$, and we call them characteristic sets. Repeating literally what was said in Subsection 2.2 we define periodic, $\mathcal{T}$-guided, and $\mathcal{T}$-proper orbits corresponding to the semigroup $\Phi_{\zeta}$. Finally we introduce the set $\mathfrak{N}_{\zeta}^{\mathcal{T}}$ of all $\mathcal{T}$-proper periodic orbits, consisting of characteristic points in $\Gamma$.

Denote by $C^{k}(\partial D)$ the set of all continuous functions on $\partial D$ whose restrictions to all sides of the triangle $D$ are $k$ times continuously differentiable functions.

Theorem 14 (cf. [2]). Assume that the characteristic sets $\mathcal{T}_{1}$ and $\mathcal{T}_{2}$ satisfy the same conditions as the guiding sets $\mathcal{T}_{1}$ and $\mathcal{T}_{2}$ in Theorem 1 . Then for any functions $F \in C(\bar{D})$ and $h \in C^{2}(\partial D)$ there is a unique generalized solution $u(x, y)$ of problem (50) if and only if the set $\mathfrak{N}_{\zeta}^{\mathcal{T}}$ is empty. The inverse operator $(F, h) \mapsto u$ is continuous: $C(\bar{D}) \times C^{2}(\partial \Omega) \rightarrow C^{2}(\bar{D})$. If $F \in C^{k}(D)$ and $h \in C^{k+2}(\partial D), k \geq 1$, then $u \in C^{k+2}(D)$ is a classical solution of the problem in question.

Proof. We restrict ourselves to the case $F=0$. Let us write down the operator $P$ in the form (49). It is clear that a linear change of variables reduces problem (50) to the problem

$$
\left(m_{1} \partial_{x}+m_{2} \partial_{y}\right) \partial_{x} \partial_{y} u=0 \quad \text { in } D, \quad u=h \quad \text { on } \partial D .
$$

Here $D$ is a domain in $\mathbb{R}^{2}$ whose boundary $\partial D$ consists of the three parts

$$
\begin{aligned}
& \Gamma_{1}=\{(x, y) \mid y=0, \quad 0 \leq x \leq 1\}, \quad \Gamma_{2}=\{(x, y) \mid x=0, \quad 0 \leq y \leq 1\}, \\
& \Gamma_{3}=\left\{(x, y) \mid x=\alpha_{1}(t), y=\alpha_{2}(t) ; \quad 0 \leq t \leq 1\right\}
\end{aligned}
$$

where

$$
\alpha_{1}(0)=0, \quad \alpha_{1}(1)=1 ; \quad \alpha_{2}(0)=1, \quad \alpha_{2}(1)=0 .
$$

(For convenience we preserve the previous notation for the domain and functions.) 
Let

$$
h=h_{1}(x) \quad \text { on } \Gamma_{1}, \quad h=h_{2}(y) \quad \text { on } \Gamma_{2}, \quad \text { and } \quad h=h_{3}(x, y) \quad \text { on } \Gamma_{3} .
$$

The continuity of the function $h$ leads to the natural compatibility conditions

$$
h_{1}(0)=h_{2}(0), \quad h_{1}(1)=h_{3}(1,0), \quad h_{2}(1)=h_{3}(0,1) .
$$

Using the postulated properties of the domain $D$ it is easy to verify that the function

$$
u(x, y)=\int_{0}^{x}\left(\int_{0}^{y} G\left(n_{1} s+n_{2} t\right) d t\right) d s+h_{1}(x)+h_{2}(y)-h_{1}(0), \quad 0 \leq x, y \leq 1,
$$

is a generalized solution to the equation in (51), satisfying boundary conditions on $\Gamma_{1} \cup \Gamma_{2}$ for any function $G \in C^{1}\left(n_{1}, n_{2}\right)$. Here $n=\left(n_{1}, n_{2}\right)$ is the unit vector orthogonal to the vector $m=\left(m_{1}, m_{2}\right)$, and $n_{1}<0, n_{2}>0$. The necessity of satisfying the boundary condition $u=h_{3}$ on $\Gamma_{3}$ leads to the following integral equation for an unknown function $G \in C(I)$ :

$$
\int_{0}^{\alpha_{1}(t)}\left(\int_{0}^{\alpha_{2}(t)} G\left(n_{1} x+n_{2} y\right) d y\right) d x=H(t), \quad 0 \leq t \leq 1 .
$$

Here

$$
H(t)=-h_{1}\left(\alpha_{1}(t)\right)-h_{2}\left(\alpha_{2}(t)\right)+h_{3}\left(\alpha_{1}(t), \alpha_{2}(t)\right)+h_{1}(0)
$$

is a given function. What is important is that the function $H(t)$, generated by an arbitrary continuous and twice piecewise differentiable function $h$ in (51), belongs to the space $\mathcal{H}(I)=C^{2} \cap C_{0}(I)$ (see Subsection 2.1). This follows from the compatibility conditions (52). The converse is also true, namely, the function $u(x, y)$ defined by (53) with $G$ a solution of equation (54) solves problem (51).

Thus, problem (51) turns out to be equivalent to the integral equation (54) which is nothing but equation (23). The unique solvability of problem (51), provided that $\mathfrak{N}_{\zeta}^{\mathcal{T}}=\emptyset$, follows directly from Theorem 1

\section{APPENDIX}

Proposition A.1. With the notation introduced in Subsection 4.1 the inequalities

$$
\alpha_{1}^{\prime}(z) \geq 0, \quad \alpha_{2}^{\prime}(z) \leq 0, \quad z \in I_{Z}
$$

hold.

Proof. It suffices to prove the first inequality.

(I) We note that if the curve $\Gamma$ in a neighborhood of a point $M\left(x_{0}, y_{0}\right) \in \Gamma$ is described by an equation $y=f(x)$ with $f$ a differentiable function, then one of the half-intervals $\left\{(x, y) \mid x=x_{0}, 0 \leq y-y_{0} \leq \varepsilon\right\}$ and $\left\{(x, y) \mid x=x_{0},-\varepsilon \leq y-y_{0} \leq 0\right\}$ is free of points of $\bar{D}$.

(II) We note also that given differentiable functions $\varphi_{1}(t)$ and $\varphi_{2}(t)$ with the same range there are points $t_{1}$ and $t_{2}$ such that

$$
\varphi_{1}\left(t_{1}\right)=\varphi_{2}\left(t_{2}\right) \quad \text { and } \quad \varphi_{1}^{\prime}\left(t_{1}\right) \varphi_{2}^{\prime}\left(t_{2}\right) \neq 0 .
$$

Indeed, localizing the problem and using an affine transformation one can reduce the proof to the case when the domains of $\varphi_{1}(t)$ and $\varphi_{2}(t)$ coincide and $\varphi_{1}^{\prime}(t)>0$. But then the result is obvious: as $t_{2}$ we take any point with $\varphi_{2}^{\prime}\left(t_{2}\right) \neq 0$, and we choose $t_{1}=\varphi_{1}^{-1} \circ \varphi_{2}\left(t_{2}\right)$. 
(III) To prove the first inequality in (A.1) assume that $\alpha_{1}^{\prime}(\tau)<0$ for some $\tau \in I_{Z}$ and take a point $\widehat{\tau}$ with $\alpha_{1}^{\prime}(\widehat{\tau})>0$. (As $\alpha_{1}(-1)=0$ and $\alpha_{1}(1)=1$, such a point certainly exists.) We choose a point $\theta \in(\tau, \widehat{\tau})$ for which

$$
A:=\alpha_{1}(\theta)=\max _{\tau \leq t \leq \widehat{\tau}} \alpha_{1}(t) .
$$

It is clear that there are positive numbers $\theta_{1}, \theta_{2}$ such that

$$
B:=\alpha_{1}\left(\theta-\theta_{1}\right)=\alpha_{1}\left(\theta+\theta_{2}\right) .
$$

But then the restrictions $\alpha_{-}(t)$ and $\alpha_{+}(t)$ of the function $\alpha_{1}(t)$ to the intervals $\left(\theta-\theta_{1}, \theta\right)$ and $\left(\theta, \theta+\theta_{2}\right)$, respectively, map their domains on $(A, B)$. In view of (II) there exist points $t^{*}$ and $t_{*}$ such that

$$
x^{*}=\alpha_{-}\left(t^{*}\right)=\alpha_{+}\left(t_{*}\right) \text { and } \alpha_{-}^{\prime}\left(t^{*}\right) \alpha_{+}^{\prime}\left(t_{*}\right) \neq 0 .
$$

We now consider three points in $\partial D$ :

$$
M_{0}=\left(x^{*}, 0\right), \quad M^{*}=\left(x^{*}, \alpha_{2}\left(t^{*}\right)\right), \quad M_{*}=\left(x^{*}, \alpha_{2}\left(t_{*}\right)\right) .
$$

For definiteness let $\alpha_{2}\left(t_{*}\right)>\alpha_{2}\left(t^{*}\right)$. Due to (A.3) each point $M^{*}$ and $M_{*}$ has a neighborhood in which $\Gamma$ is described by an equation

$$
x_{2}=f\left(x_{1}\right), \quad\left|x-x^{*}\right|<r,
$$

with $f=\alpha_{2} \circ \alpha_{1}^{-1}$. By virtue of (I) on the straight line $x_{1}=x^{*}$ a half-neighborhood of each point $M^{*}$ and $M_{*}$ is free of points of $D$. As $M_{0}$ and $M^{*}$ belong to $\bar{D}$ and $\bar{D}$ is $\boldsymbol{l}_{2}$-convex, the line segment $M_{0} M^{*}$ lies wholly in $\bar{D}$. But this contradicts what has been said about the point $M_{*}$. This completes the proof of Proposition A.1.

Proof of Proposition 10. It is clear that

$$
\operatorname{dim} \operatorname{ker}(R-\mathcal{N})=\operatorname{dim}\left(E-R^{-1} \mathcal{N}\right)<\infty
$$

since $R-\mathcal{N}=R\left(E-R^{-1} \mathcal{N}\right)$. Denote $R-\mathcal{N}=P$ and $E-R^{-1} \mathcal{N}=Q$ so that $P=R Q$. By definition

$$
\operatorname{coker} Q=B / \mathcal{R}(Q)=\{f+\mathcal{R}(Q)\} \text {,5 }
$$

where $f+\mathcal{R}(Q)$ denotes a class of elements $f^{\prime}$ in $B$ such that $f-f^{\prime} \in \mathcal{R}(Q)$. Therefore

$$
\text { coker } P=R B / \mathcal{R}(R Q)=\{R f+\mathcal{R}(R Q)\} .
$$

But $R B=B$ due to the invertibility of $R$ and $R f-R f^{\prime} \in \mathcal{R}(R Q)$ if and only if $f-f^{\prime} \in \mathcal{R}(Q)$. This means that

$$
\operatorname{dim} \text { coker } P=\operatorname{dim} \operatorname{coker} Q .
$$

It remains to combine the latter equality with (A.4) and to use the compactness of the operator $R^{-1} \mathcal{N}$ and the F. Riesz-Schauder theorem 6

\footnotetext{
${ }^{5} \mathcal{R}(Q)$ denotes the range of the operator $Q$.

${ }^{6}$ This theorem states: if $E$ and $\mathcal{N}$ are identical and compact operators (respectively) in a Banach space $B$, then ind $(E-\mathcal{N})=\operatorname{dim} \operatorname{ker}(E-\mathcal{N})-\operatorname{dim} \operatorname{coker}(E-\mathcal{N})=0$.
} 
Proof of Proposition 11. It suffices to prove the convergence of the series $\sum_{k=0}^{\infty}\left\|R^{k}\right\|$. Let $\|T\|=\tau$ and $\left\|T^{m}\right\|=\gamma<1$. Then for all integers $p=1, \ldots, m-1$ the inequality $\left\|T^{n m+p}\right\| \leq \tau^{p} \gamma^{n}$ holds. This implies

$$
\sum_{k=0}^{\infty}\left\|R^{k}\right\|=\sum_{n=0}^{\infty} \sum_{p=0}^{m-1}\left\|T^{n m+p}\right\| \leq\left(\tau^{m}-1\right) /(\tau-1)(1-\gamma) .
$$

\section{ACKNOWLEDGEMENTS}

The results and the methods represented in this paper were discussed at different times with my colleagues and friends Professors G. Belizkii, J .Bernstein, Yu. Lyubich, L. Volevich, and A. Vinogradov. I would like to express to all of them my hearty thanks.

\section{REFERENCES}

[1] I.Gel'fand, M.Graev and N.Vilenkin, Integral Geometry and Representation Theory, New York, Academic Press, 1966. MR 34:7726

[2] B.Paneah, On a Problem in Integral Geometry Connected to the Dirichlet Problem for Hyperbolic Equations, IMRN International Mathematics Research Notices, 1997, No.5, 213-222. MR 98k:53097

[3] B.Paneah, A Problem in Integral Geometry with Applications to the Dirichlet Problem for a Class of Hyperbolic Differential Equations, Differential Equations, Asymptotic Analysis and Mathematical Physics, Math.Res. 100, Akademic-Verlag, Berlin, 1997, 256-261. MR 98h:53115

[4] B.Paneah, On Solvability of Functional Equations Connected with Dynamical Systems with Two Generators, Funct. Anal. Appl. 37, No.1, pp 46-60, 2003.

[5] B.Paneah, Noncommutative Dynamical Systems with Two Generators and Their Applications in Analysis, Discrete and Continuous Dynamical Systems (DCDS), v. 9, No. 6, pp. 1411-1422, 2003.

Department of Mathematics, Technion, Haifa, 32000, Israel

E-mail address: peter@tx.technion.ac.il 